\title{
UPSLOPE WALKING WITH TRANSFEMORAL PROSTHESIS USING OPTIMIZATION BASED SPLINE GENERATION
}

\author{
A Thesis \\ by \\ VICTOR CHRISTIAN PAREDES CAUNA
}

\author{
Submitted to the Office of Graduate and Professional Studies of \\ Texas A\&M University \\ in partial fulfillment of the requirements for the degree of \\ MASTER OF SCIENCE
}

\begin{abstract}
Chair of Committee, Pilwon Hur
Committee Members, Bryan Rasmussen

Shuguang Cui

Head of Department, Andreas A. Polycarpou
\end{abstract}

May 2016

Major Subject: Mechanical Engineering

Copyright 2016 Victor Christian Paredes Cauna 


\begin{abstract}
Powered prosthetic devices are robotic systems that are aimed to restore the mobility of subjects with amputations above the knee by imitating the behavior of a normal human leg. Powered prostheses have diverse advantages compared to passive devices, including the possibility of reducing the metabolic cost of the user, providing net power into the walking gait and walking on diverse terrain. In particular, this thesis is focused on the capacity of powered transfemoral prostheses to adapt to diverse terrains. Since most terrains consist of flat and inclined surfaces, it is important that a transfemoral prosthesis can walk on these surfaces and have the capacity to seamlessly transition from one surface to the another. However, currently available controllers require either intention recognition procedures that delay the terrain transition or a collection of parameters that require a large tuning process for each possible surface profile. In this thesis, we propose a framework that can generate automatically stable and human-like gaits for both surfaces with immediate transition between them. The new framework is based on human-inspired control and a spline-based trajectory generation. Specifically, the proposed method i) inserts a set of cubic splines that smoothly blend the flat ground joint trajectories into arbitrary upslope surface joint trajectories for the ankle and knee joints and ii) employs a low gain PD control for terrain adaptation for various unknown surfaces. This framework is implemented on the powered transfemoral prosthetic device, AMPRO II, for both flat ground and upslope walking to test its use as a nominal controller. The experimental results confirm that the proposed framework provides walking gaits for flat ground and upslope with seamlessly smooth transitioning gaits between them.
\end{abstract}




\section{DEDICATION}

I would like to remember always the blessings from God that accompanied me through all my life and in particular during grad school, this work is dedicated to him. Also, I wish to dedicate this work to my parents. Without their support, none of my academic formation would be possible. Struggles never stopped their love and support for me. 


\section{ACKNOWLEDGEMENTS}

This work is motivated by my supportive and patient advisor Dr. Pilwon Hur. I want to acknowledge as well the opportunity to work under his guidance in rehabil-

itation abd robotics. Also, I want to extend the acknowledgment to all the people in HUR Group that generated a positive learning environment. 


\title{
NOMENCLATURE
}

\author{
AMBER A\&M Bipedal Experimental Robot \\ AMPRO A\&M Prosthetics \\ CAN Controller Area Network \\ EKF Extended Kalman Filter \\ IMU Inertial Measurement Unit \\ PD Proportional and Derivative \\ QP Quadratic Program \\ CLF Control Lyapunov Function \\ CWF Canonical Walking Functions \\ ECWF Extended CWF \\ KKT Karush-Kuhn-Tucker Condition \\ HZD Hybrid Zero Dynamics \\ PHZD Partial Hybrid Zero Dynamcics \\ $\mathrm{PZ}_{a} \quad$ Surface Generated due to PHZD with Parameter $\alpha$ \\ HIO Human-Inspired Optimization \\ HIC Human-Inspired Control \\ SHIC Spline-based HIC \\ HD Human Data \\ HIC Human-inspired control \\ SHIC Splines-based human-inspired control
}




\section{TABLE OF CONTENTS}

Page

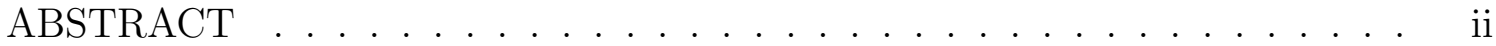

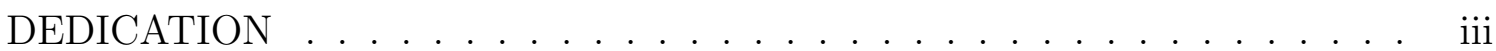

ACKNOWLEDGEMENTS .................... . . iv

NOMENCLATURE ....................... . . . . .

TABLE OF CONTENTS ............................ vi

LIST OF FIGURES . . . . . . . . . . . . . . . . . . viii

LIST OF TABLES ....................... . . . . . . . . . .

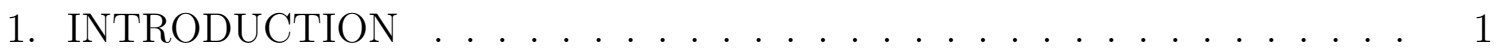

1.1 Upslope Walking . . . . . . . . . . . . . . . . . . 6

1.2 Convex Optimization for Upslope Walking . . . . . . . . . . . . 7

1.3 AMPRO II . . . . . . . . . . . . . . . . . . . . . . 8

1.4 Thesis Structure. . . . . . . . . . . . . . . . . 10

2. HUMAN UPSLOPE WALKING ANALYSIS . . . . . . . . . . . . . . . . 12

2.1 Bipedal Locomotion _. . . . . . . . . . . . . . . . . . . . 12

2.1.1 Kinematics of Human Walking . . . . . . . . . . . . . . . 12

2.2 Upslope Walking Kinematics . . . . . . . . . . . . . . . . . . . . . . . 15

2.3 Human Walking Data . . . . . . . . . . . . . . . . . . 15

2.4 Strategies for Upslope Walking . . . . . . . . . . . . . . . 17

2.4.1 Human Strategy . . . . . . . . . . . . . . . . . . 17

2.4.2 Controller Strategy . . . . . . . . . . . . . . . 18

3. FLAT TERRAIN GAIT GENERATION . . . . . . . . . . . . . . . . 20

3.1 Bipedal Model . . . . . . . . . . . . . . . . . . . 20

3.2 Human-Inspired Control (HIC) . . . . . . . . . . . . . . . 24

3.2.1 Canonical Walking Functions (CWF) . . . . . . . . . . . . 24

3.2.2 Human-Inspired Outputs (HIO) . . . . . . . . . . . . 26

3.2.3 Control Design . . . . . . . . . . . . . . . 27 
3.2.4 Partial Hybrid Zero Dynamics . . . . . . . . . . . . . . . . . 28

3.3 Human-Inspired Optimization (HIO) $\ldots \ldots \ldots \ldots$

3.4 Motion Capture System . . . . . . . . . . . . . . . . . . 30

3.5 Simulation of the Flat Ground Gait . . . . . . . . . . . . . . 33

3.6 Gait Generation for Non-Flat Terrain . . . . . . . . . . . . . . . . 34

3.6.1 Extended Canonical Walking Functions (ECWF) for Rough Terrain . . . . . . . . . . . . . . . . 34

3.6 .2 Upstairs Walking . . . . . . . . . . . . . . . 35

3.6 .3 Limitations . . . . . . . . . . . . . . . . . . . . . 35

4. OPTIMIZATION-BASED SPLINE GENERATION FOR UPSLOPE WALK-

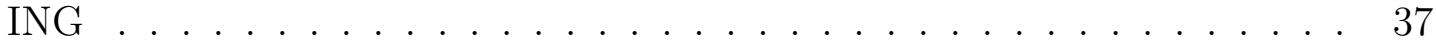

4.1 Problem Formulation . . . . . . . . . . . . . . . . . . . . . 37

4.2 Analytical Solution . . . . . . . . . . . . . . . . . . . 44

4.3 Human-Inspired Walking with Cubic Splines . . . . . . . . . . . . 45

4.3 .1 Generated Trajectories . . . . . . . . . . . . . . 46

4.4 Implementation Details . . . . . . . . . . . . . . . . . . . . 47

5. RESULTS . . . . . . . . . . . . . . . . . . . . . 49

5.1 Flat Ground Walking . . . . . . . . . . . . . . . 50

$5.1 .1 \quad$ HIC Results . . . . . . . . . . . . . . . . . . . 51

$5.1 .2 \quad$ SHIC Results . . . . . . . . . . . . . . . . . 52

5.2 Upslope Walking . . . . . . . . . . . . . . . . . . . . . . 52

5.3 Controllers Comparison . . . . . . . . . . . . . . . . . . . 52

5.3.1 Flat Ground Walking using HIC and SHIC . . . . . . . . . 53

5.3.2 Flat Ground and Upslope Walking using SHIC . . . . . . . . . 54

5.4 SHIC Control Action . . . . . . . . . . . . . . . . . . 54

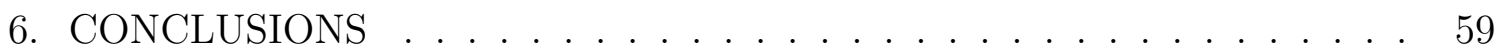

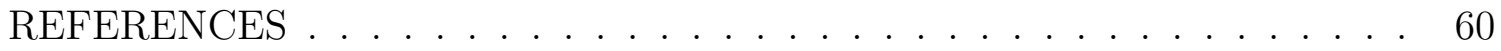




\section{LIST OF FIGURES}

FIGURE

Page

1.1 Ottobock 3R60 EBS knee joint with a prosthetic foot. This passive prosthesis is controlled through a hydraulic system adapted to the damping requirements of human walking. . . . . . . . . . . . 3

1.2 Ottobock's C-Leg is an state-of-the-art microprocessor controlled knee. This powered prosthesis is also controlled through an hydraulic system with controlled damping. . . . . . . . . . . . . . . . . . . . .

1.3 The Vanderbilt prosthesis is a powered transfemoral device, developed at the Center of Intelligent Mechatronics in Vanderbilt University. . . 4

1.4 AMPRO I, a powered transfemoral prosthesis based on the HIC. . . . 6

1.5 AMPRO II, the second generation of prosthetic devices at Texas A\&M. The main components are listed. . . . . . . . . . . . . . . .

1.6 IMU placement on the subject. The IMUs are used to interface with AMPRO II . . . . . . . . . . . . . . . . . . . .

2.1 Illustration of the phases of human walking under the assumption of flat foot walking. During double support phase both legs supports the body weight contrary to single support phase where one the legs support the body weight. . . . . . . . . . . . .

2.2 The ankle joint can move in two directions, to avoid ambiguity those directions are called dorsiflexion and plantar flexion. In the case of the knee the directions of rotation are called flexion and extension. . .

2.3 Ankle trajectory for $0^{\circ}, 5^{\circ}, 8^{\circ}$ and $10^{\circ}$ slope inclinations. The data is taken from McIntosh $[16]{ }^{1} \ldots \ldots \ldots \ldots \ldots$

2.4 Knee trajectory for $0^{\circ}, 5^{\circ}, 8^{\circ}$ and $10^{\circ}$ slope inclinations. The data is taken from McIntosh $[16]{ }^{1} \ldots \ldots \ldots \ldots \ldots$

2.5 Low gain PD action used to enable terrain adaptation. . . . . . . . 18 
2.6 Typical ankle and knee flat ground trajectories. The red shaded region represents the action of the splines and the blue shaded region the action of the low PD control. ...................

3.1 Bipedal robotic model considering the same anthropomorphic dimensions as the human subject. . . . . . . . . . . . . . . . .

3.2 Human data collected though IMUs represented as canonical walking functions. They are conformed by the hip position $(\mathrm{m})$ and the joints relations $(\mathrm{rad}) . \ldots \ldots \ldots . \ldots \ldots 25$

3.3 Trajectories captured by the IMU-based motion system for ankle and knee. . . . . . . . . . . . . . . . . . . 31

3.4 Trajectories generated by the human-inspired optimization for ankle and knee. . . . . . . . . . . . . . . . . . . . 32

3.5 Ankle and knee phase portrait, indicating a continuous evolution of the system and a discrete change in velocity at impact. . . . . . . . . 32

3.6 Canonical walking functions obtained in the simulation. . . . . . . . . 33

3.7 Gait tile simulation using feedback linearization control. . . . . . . . 34

4.1 Two disconnected trajectories $C 1$ and $C 2$ can be connected trough a trajectory $S$, starting from time $t_{s}$ and finishing on $t_{c} \ldots . . . .37$

4.2 Connecting two different trajectories using a set of splines joined by specific waypoints . . . . . . . . . . . . . . . . . 39

4.3 Cubic splines connected through waypoints . . . . . . . . . . . . . 39

4.4 Trajectory generation for ankle and knee joint. The splines has been generated in two extremes to show the effect of the splines. . . . . . 47

5.1 Healthy subject using a knee adapter to use the transfemoral prosthesis AMPRO II . . . . . . . . . . . . . . . . . . . . . . . . . . . . . 49

5.2 Indoors flat ground walking experiment using SHIC . . . . . . . . . . 50

5.3 Outdoors experiment using SHIC . . . . . . . . . . . . 51

5.4 Ankle and knee tracking for flat ground walking using HIC. . . . . . 51

5.5 Ankle and knee tracking for flat ground walking using SHIC. . . . . . 52 
5.6 Upslope walking trajectories for ankle and knee. The slope inclination

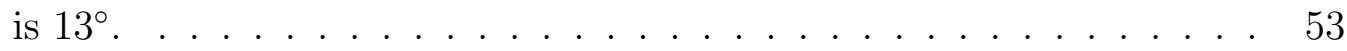

5.7 Superposition of the trajectories for flat ground surfaces using both controllers: HIC (red) and SHIC (green) . . . . . . . . . . 53

5.8 2-steps trajectory differences between flat ground (green) and upslope walking (red) using SHIC. . . . . . . . . . . . . . . 54

5.9 SHIC low gain PD (red region) and spline generation (blue region) for

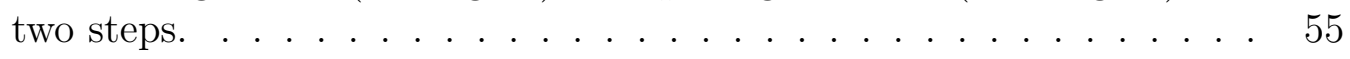

5.10 Flat ground walking using SHIC. . . . . . . . . . . 56

5.11 Transition between flat ground to upslope walking using SHIC. . . . 57

5.12 Upslope walking sequence using SHIC . . . . . . . . . . . 58 


\section{LIST OF TABLES}

TABLE

Page

4.1 Implementation parameters $\ldots \ldots \ldots \ldots \ldots \ldots$ 


\section{INTRODUCTION}

An amputation can play a negative role in the development of the persons affecting the access to some opportunities and even impacting on their depression levels [17]. In particular, lower limb amputation also imposes a big impact on the mobility, specifically walking gait. This motivates the development of prostheses that can attenuate this problem by imitating the behavior of normal human legs and providing the possibility of recovering the damaged walking skills.

Lower limb amputations can be classified as amputations below the knee (transtibial) and above the knee (transfemoral). Experiments suggest that transfemoral amputees behave in a less active life style compared to transtibial amputees. Particularly the maximum aerobic capacity was significantly lower in transfemoral amputees compared to transtibial amputees [24]. These facts justify the study and development

of transfemoral devices to bypass the limitations of transfemoral amputees. Transfemoral prostheses are expected to achieve a human-like walking and meet several requirements, such as bearing the user's body weight, being safe on its operational cycle, cooperating with the user, providing possibility to traverse diverse terrains, etc.

It is estimated that there are approximately 185,000 new amputations each year in the United States [15]. Furthermore, according to a report by the National Center for Health Statistics it is estimated that from the 1.2 million people in the United States that lives with limb loss, 18.5 percent are trans-femoral amputees [21]. Despite the long history of feedback control, most of the available prostheses in the market are energetically-passive devices. In other words, they do not contribute to the insertion of net power during the walking cycle. Essentially they are 
mechanically-actuated devices with passive elements such as springs and dampers. Consequently, their mechanical characteristics are fixed and the range of terrains they can traverse is very limited. However, even with the exposed limitations of passive transfemoral prosthesis, they are reliable, lightweight and easy to use.

In recent years, several powered devices have been developed to overcome some of the weaknesses that passive prostheses have. These weaknesses include, for instance, the lack of user's control over the device and the limited adaptation to different kind of terrains. Basically a powered transfemoral device is composed by ankle and knee actuators driven by a computer implementing a feedback control algorithm designed to interact with the user and resemble the human walking gait for different scenarios. For example, the Ottobock's 3R60 EBS (Fig 1.1) and the C-Leg4 (1.2) are commercially-available microcontroller-based knee devices that actuate only over the knee. The microcontroller changes the knee damping properties depending on the selected task.

Powered transfemoral prostheses with actuation in both ankle and knee can offer the possibility to enhance the performance of the walking gait compared to the knee-actuated devices. Although it increases the complexity of the system, advances in feedback control can allow the generation of human-like gaits with on-board processing. 


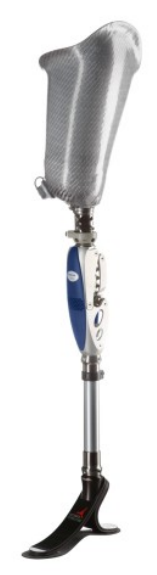

Figure 1.1: Ottobock 3R60 EBS knee joint with a prosthetic foot. This passive prosthesis is controlled through a hydraulic system adapted to the damping requirements of human walking.

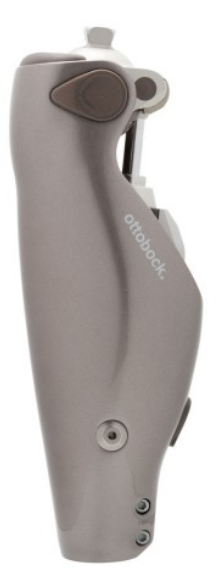

Figure 1.2: Ottobock's C-Leg is an state-of-the-art microprocessor controlled knee. This powered prosthesis is also controlled through an hydraulic system with controlled damping. 
In the research community, one of the most representative fully-actuated transfemoral prosthesis was developed by Vanderbilt University [11, 22]. This prosthesis is an electrically-actuated device controlled by an impedance control framework, where the impedance is modeled as a linear spring and a damper that change their properties along the gait cycle [8]. This cycle is decomposed into several phases along the gait progression. Each phase is represented by a unique set of impedance parameters which are selected through a finite state machine. These parameters defining the impedance must be tuned offline based on joint sensor data, video recordings and user feedback [22]. The Vanderbilt prosthesis is shown in Fig. 1.3. Note that this framework depends on an offline tuning process to acquire the impedance parameters for each subject, which limits its practical implementation for a massive number of users.

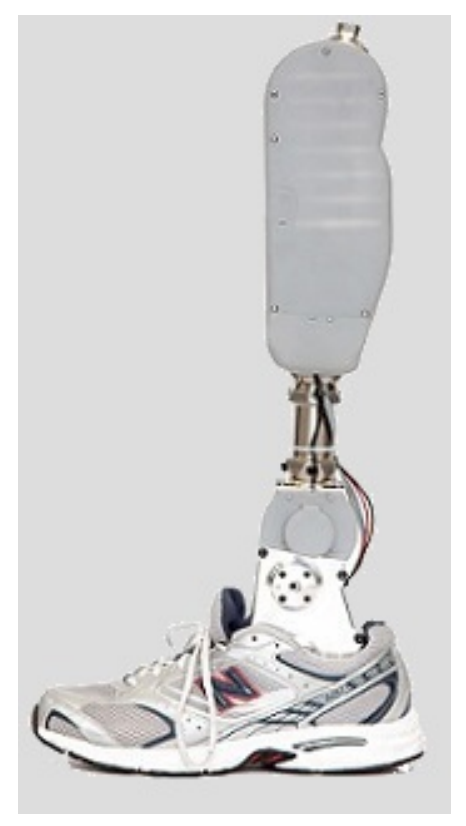

Figure 1.3: The Vanderbilt prosthesis is a powered transfemoral device, developed at the Center of Intelligent Mechatronics in Vanderbilt University. 
An important method that does not require a tuning process to generate a walking gait is the Human-Inspired Control (HIC) $[2,1]$. It is a control framework based on Hybrid Zero Dynamics (HZD) [27, 26] that was originally formulated for bipedal robotic walking. HZD combines discrete events (i.e., impact at the heel strike) and the continuous dynamics in the context of hybrid systems [20]. HIC [1, 2] generates stable and human-like walking gaits using human walking data and an HZD formulation. This framework is based on the Human-Inspired Optimization (HIO) where human walking data is fitted by several lower-dimensional motion primitives. This method has successfully been implemented in bipedal robots such as AMBER I [28], AMBER II [30], DURUS [7] and ATRIAS [6].

The HIC framework has been extended for powered transfemoral prosthesis and tested in the prosthetic device AMPRO I [33] (Fig. 1.4), the first generation of transfemoral prosthesis developed by AMBER Lab (now at Georgia Tech) at Texas A\&M University. Two different assumptions on the walking gait can be considered under the HIC framework: i) a flat foot and single-contact behavior that captures the minimum essentials of human walking [33], and ii) a multi-contact behavior that expands the results on the flat foot assumption and that generates a more humanlike walking gait [31]. The assumption of flat foot walking is useful for testing new algorithms since it provides a simple framework for implementation, after a successful implementation of an algorithm it can be translated into the multi-contact framework. It is reported that the knee trajectory in a flat foot framework was similar to real human knee trajectory, whereas the simulated ankle trajectory in a flat foot framework was significantly different from the real human ankle trajectory [33]. However, this deviation was mitigated by using multi-contact framework because multi-contact framework includes more phases of the human walking cycle [31]. 

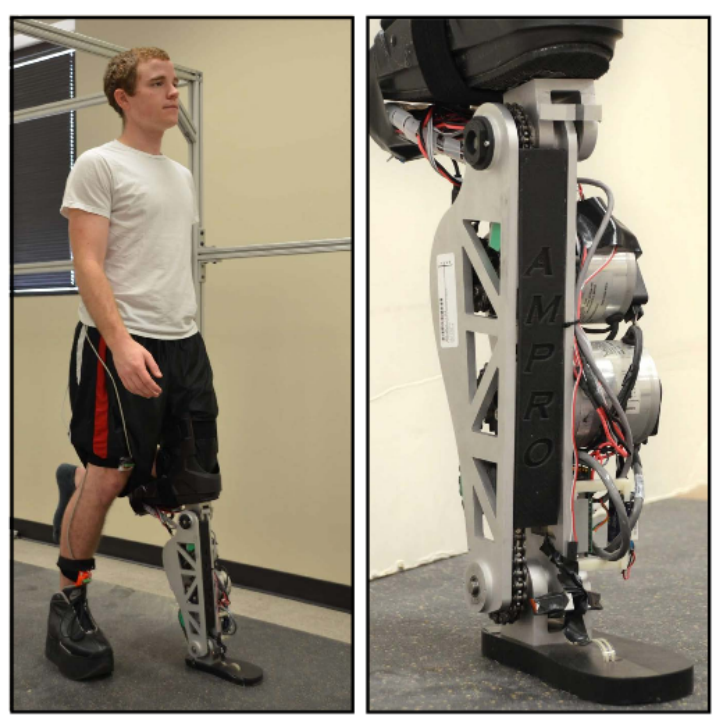

Figure 1.4: AMPRO I, a powered transfemoral prosthesis based on the HIC.

\subsection{Upslope Walking}

As mentioned earlier, an important characteristic required for prosthesis is the capacity to traverse diverse terrains. To achieve this with a powered transfemoral prosthesis, it is important to construct controllers able to traverse common terrains in everyday life, such as rough terrain, stairs and ramps. Given that flat ground and sloped surfaces are very common for most environments, this thesis focuses on the generation of walking gaits for flat ground and upslope surfaces.

Even though many research efforts have been placed in flat ground walking [5, 33, 22], the work of [23] extended the previous flat ground algorithm based on impedance control to inclined surfaces. The algorithm involves an offline tuning process to get the impedance parameters for two different inclinations of $5^{\circ}$ and $10^{\circ}$. When an inclined surface is detected while walking, the closest inclination angle (either $5^{\circ}$ or $10^{\circ}$ ) from the estimated inclination angle is determined and used for the impedance 
parameters. However, as the number of inclinations on the database increase, the tuning process becomes tedious and infeasible.

As indicated earlier, HIC automatically generates stable and human-like walking gaits for transfemoral prosthesis, bypassing the tuning process. In the literature, HIC has been used for a ascending stair walking algorithm and implemented in AMPRO I [29]. This algorithm used a priori information about the stairs profile (i.e., step height and width). Since HIC involves HIO, this algorithm cannot be computed online due to computational load required to solve the optimization.

Furthermore, information about the terrain cannot be obtained in advance for most situations, running HIO for many unknown surfaces and subjects beforehand seems impractical. This limitation of HIC motivates the usage of more flexible schemes. However, it is still beneficial to keep the advantages of HIC, specially the automatic generation of walking gaits.

To generate upslope walking trajectories without the burden of running HIO for every possible slope, it is possible to use a convex optimization formulation because of its fast convergence to a solution that can be performed in real time. The generation of trajectories based on a convex optimization formulation has been utilized in legged robotics with the use of cubic splines [10].

\subsection{Convex Optimization for Upslope Walking}

Since humans can walk on sloped surface without any problems, it would be beneficial to get insights from human upslope walking. That is, comparing joint kinematics during upslope walking and during flat ground walking may give us directions about qualitative strategies used by humans traversing this kind of terrains. The main idea is the generation of upslope walking trajectories by blending nominal flat ground walking gaits using the strategies found by observing human data. 
Considering biomechanical characteristics of walking on inclined surfaces, [16] reported that the knee seems to be sensitive enough to differentiate the surface inclination angle. Also, the knee flexion and ankle dorsiflexion increase as the surface slope increases. It suggests that the ankle and knee present some trends on its evolution with respect to the flat ground walking gait. These trends will be analyzed for the generation of strategies in the prosthetic device.

\section{$1.3 \quad$ AMPRO II}

AMPRO (A\&M Prosthetic) II is the second version of the AMPRO prostheses series that are custom-designed and built at Texas A\&M University by AMBER Lab (now at Georgia Tech). Compared to the first generation of AMPRO [33], the main improvements of the second version are three folds. a) The weight is reduced to $5 \mathrm{~kg}$, which is $3 \mathrm{~kg}$ lighter than AMPRO I. b) The height is reduced by $71 \mathrm{~mm}$ and the width is reduced by $36 \mathrm{~mm}$. The smaller size allows a wider range of subject height. c) The motors and electronics are placed higher up on the calf, resulting a higher center of mass position, which yields a smaller centroidal moment of inertial for the user. Additionally, two FlexiForce force sensors are mounted on the heel and toe for contact detection. The main code structure including the online gait generation code is running on a low-powered single core micro computer, BeagleBone Black, at $200 \mathrm{~Hz}$. Details of the design diagram can be seen in Fig. 1.5. In order to interface with AMPRO II, the human leg is instrumented with two IMUs located at the shin and thigh (Fig. 1.6). 


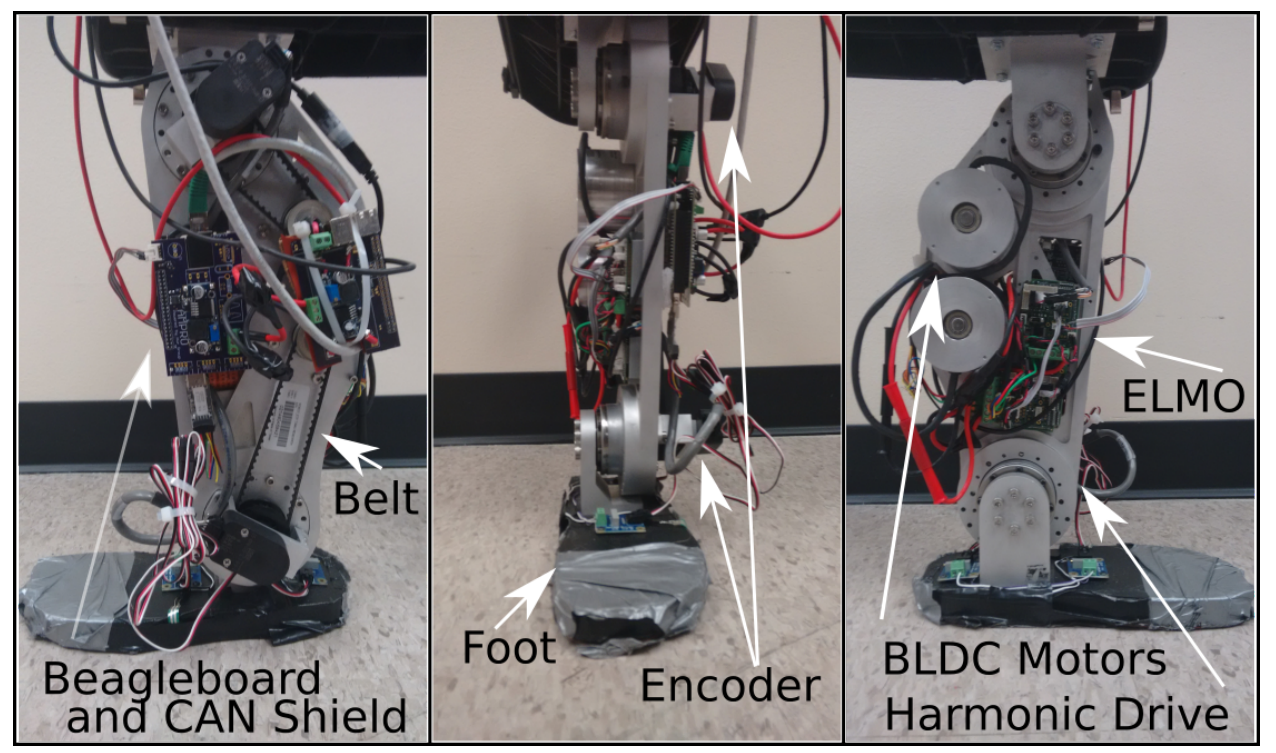

Figure 1.5: AMPRO II, the second generation of prosthetic devices at Texas A\&M. The main components are listed.

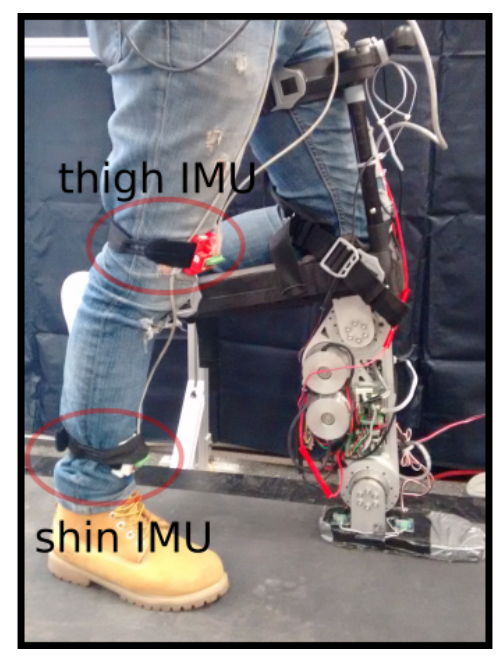

Figure 1.6: IMU placement on the subject. The IMUs are used to interface with AMPRO II 
The HIC is implemented as a high level controller on the embedded BeagleBone Black, which takes as inputs the values from the encoders, the FlexiForce sensors and the IMUs data. The desired torques are commanded to the motor drivers (ELMO) through CAN communication and each motor driver implements a low-level joint controller to deliver the desired torque to their respective joints. To process the IMU data, another BeagleBone Black is used to compute an extended Kalman filter (EKF) which estimates the human hip position that is used as a parameterization of time for the human-inspired control.

\subsection{Thesis Structure}

The flow of the thesis is as follows. In Chapter 2 the underlying kinematics for upslope walking are studied to characterize the strategies that healthy adults use when they are traversing inclined surfaces. By comparing ankle and knee trajectories from flat ground to upslope walking, it is possible to draw important conclusions about how humans blend the flat ground trajectories into upslope trajectories.

In Chapter 3, there is a presentation of the basic definitions of the human walking and a summary of the HIC along with some discussion about its advantages and limitations in the context of upslope walking.

The convex optimization formulation for the cubic splines is explained and constructed in Chapter 4. This formulation is based on the analysis of the human strategies for upslope walking. This chapter also shows the modification of canonical walking functions to take consideration of the generated splines.

The results of the application of the cubic splines with the HIC are shown in Chapter 5 . The blended trajectories designed using the spline formulation are shown and explained for different scenarios. The implementation and data acquisition were conducted using the prosthetic device AMPRO II. 
The conclusions are presented in Chapter 6. Additionally, a comparison is made between the standard walking controller for flat ground terrain and the spline-based controller. The idea is to motivate the usage of the spline-based controller as a general framework to traverse different surfaces within the same framework. Finally, open questions and potential improvements are discussed for guiding new research on this topic. 


\section{HUMAN UPSLOPE WALKING ANALYSIS}

This chapter presents an overview of the human data analysis for flat ground and upslope walking gaits. In particular, by analyzing the kinematic data of healthy humans during upslope walking it is expected to extract high level strategies that makes possible the main formulation: blending flat ground trajectories into upslope walking gaits.

\subsection{Bipedal Locomotion}

Bipedal locomotion is a highly non-linear process, several muscles can contribute during a single movement of a limb, in consequence the analysis of the dynamics and forces involved during human walking can be complex. Given the difference in dynamics between a human leg and a transfemoral prosthesis, it is not possible to

find a simple correspondence between forces, instead a kinematic relation can be used to capture the high-level human behavior.

\subsubsection{Kinematics of Human Walking}

As explained human walking can be studied in the light of kinematics despite the differences in dynamics between a robotic model and an actual human. To study the kinematics of human walking it is important to distinguish between the basic walking phases that are involved in the walking cycle: single support phase and double support phase. During single support phase (Fig. 2.1) the leg supporting the human body weight is called stance leg and the leg moving forward is the swing leg. Furthermore, in the context of prostheses, it is useful to differentiate between the human leg and the prosthetic leg by making explicit the leg performing an action, for instance, it is useful to use human stance leg or prosthesis stance leg to differentiate between those 
outcomes.

During walking, there is a cyclic process on the movement of the legs, starting in double support phase (Fig. 2.1), where both legs support the human body weight, and then single support phase, where one of the legs become stance leg and the other swing leg. The swing legs moves to its desired location until it impacts with the ground, entering again to the double support phase, finalizing one step. The next step repeats the cycle after swapping the naming of the legs (stance leg and swing leg). Note that for the scope of the thesis, flat foot walking is being imposed on the walking gait and behaviors such as heel strike or toe push are left for future work.

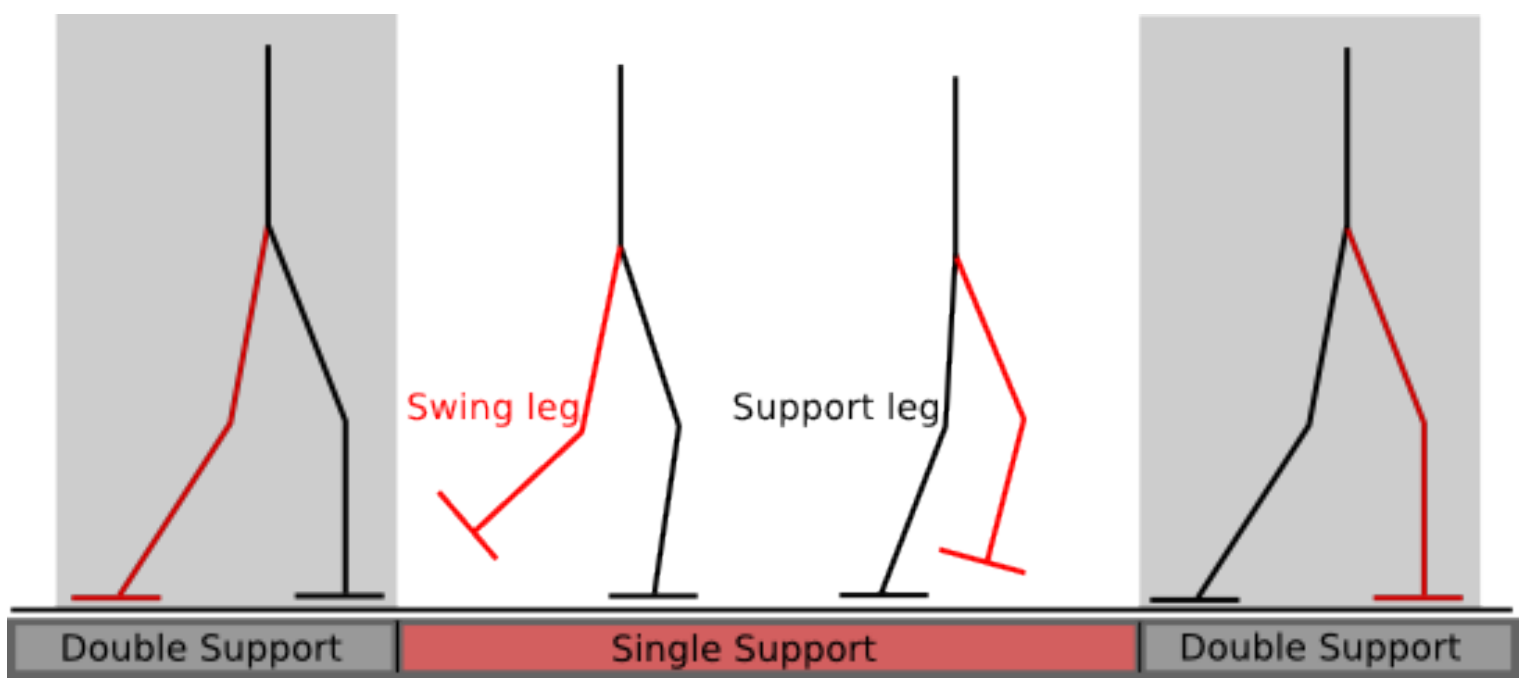

Figure 2.1: Illustration of the phases of human walking under the assumption of flat foot walking. During double support phase both legs supports the body weight contrary to single support phase where one the legs support the body weight.

The knee and ankle movements can be described by their direction of movement, particularly flexion and extension plays an important role to identify unambiguously 
the direction of movement of a joint. For ankle, dorsiflexion happens when the foot rotates around the ankle toward the leg and plantarflexion otherwise (Fig. 2.2). In the case of the knee, extension happens when this rotation makes the leg move forward and knee flexion otherwise (Fig. 2.2).

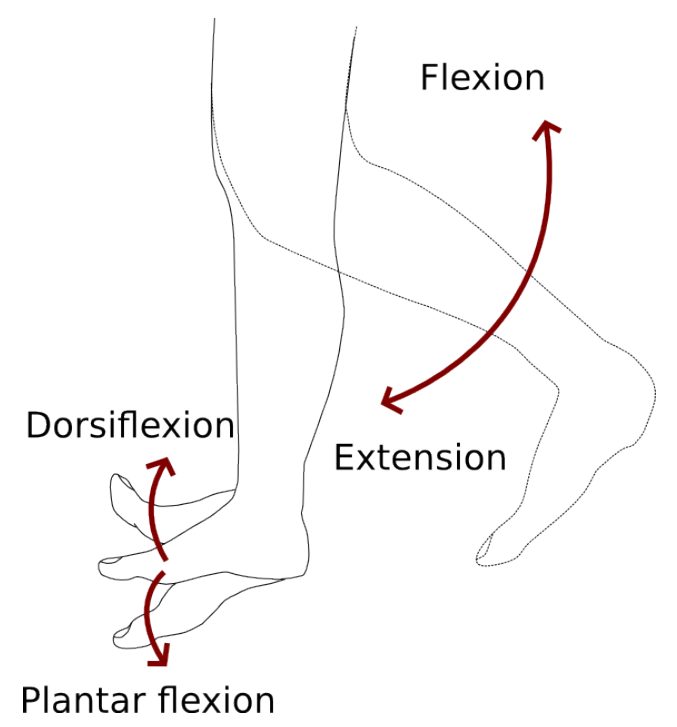

Figure 2.2: The ankle joint can move in two directions, to avoid ambiguity those directions are called dorsiflexion and plantar flexion. In the case of the knee the directions of rotation are called flexion and extension.

This subsection has described important terminology to reference rotational movements of the lower limbs that will be useful for future references in the thesis. The next subsection will explore the kinematics involving upslope walking in healthy humans. 


\subsection{Upslope Walking Kinematics}

Inclined surfaces are often traversed during locomotion activities. Pedestrians walk on this kind of surface frequently in the streets, buildings and other envi-

ronments. In consequence slope walking is an important terrain that needs to be traversed by persons using transfemoral prostheses. As a first step, this thesis aims to design upslope walking gaits by looking at human data, that would allow to collect important conclusions of upslope walking.

A great amount of study has focused primarily on flat ground walking [16], studying the changes in the kinematics pattern with respect to flat ground walking when humans perform upslope walking may expose some inherent behavior that can provide insight into the control strategies used [12] by humans traversing inclined surfaces. Those strategies could be captured and implemented into prosthetic devices in order to generate upslope walking gaits from a suitable nominal flat ground walking gait.

\subsection{Human Walking Data}

Based on the studies in inclined surface walking $[16,12,14]$, it is shown that the knee flexion increases as the inclination of the surface increases. In particular, the knee trajectory for different surfaces (Fig. 2.4) indicates a consistent trend on the trajectory changes with respect to slope increase. The same trend, although with less variation, is present in the ankle trajectories (Fig. 2.3). This increase in the initial and final points of the trajectories for both joints are explained partially on the geometry of the surface because it yields the ankle dorsiflexion and knee flexion to increase in order to avoid foot scuffing with the inclined surface. 


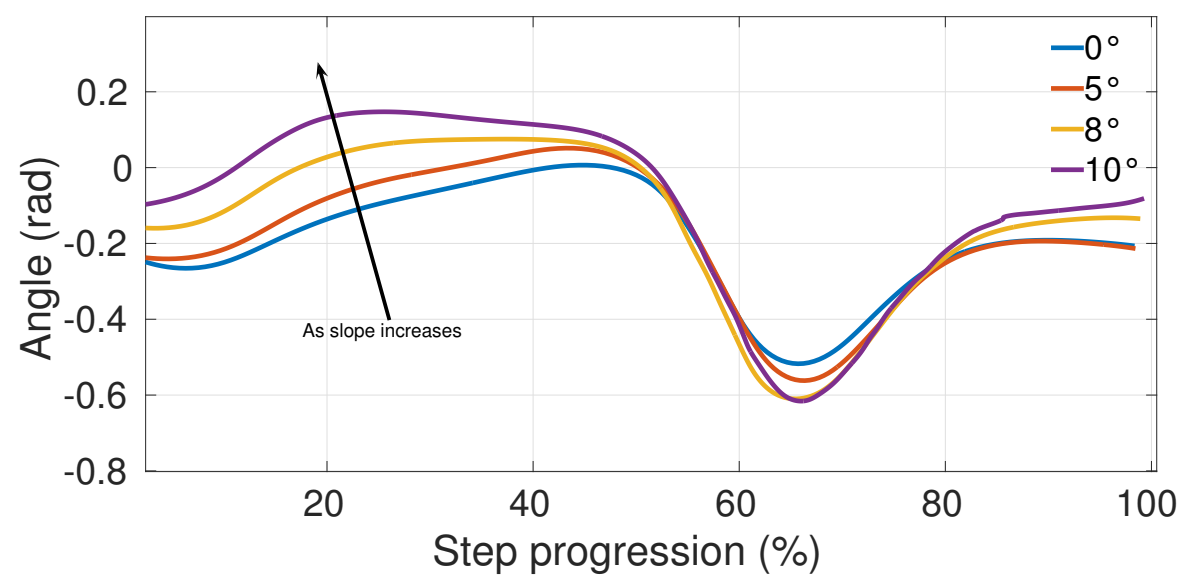

Figure 2.3: Ankle trajectory for $0^{\circ}, 5^{\circ}, 8^{\circ}$ and $10^{\circ}$ slope inclinations. The data is taken from McIntosh [16]. ${ }^{1}$

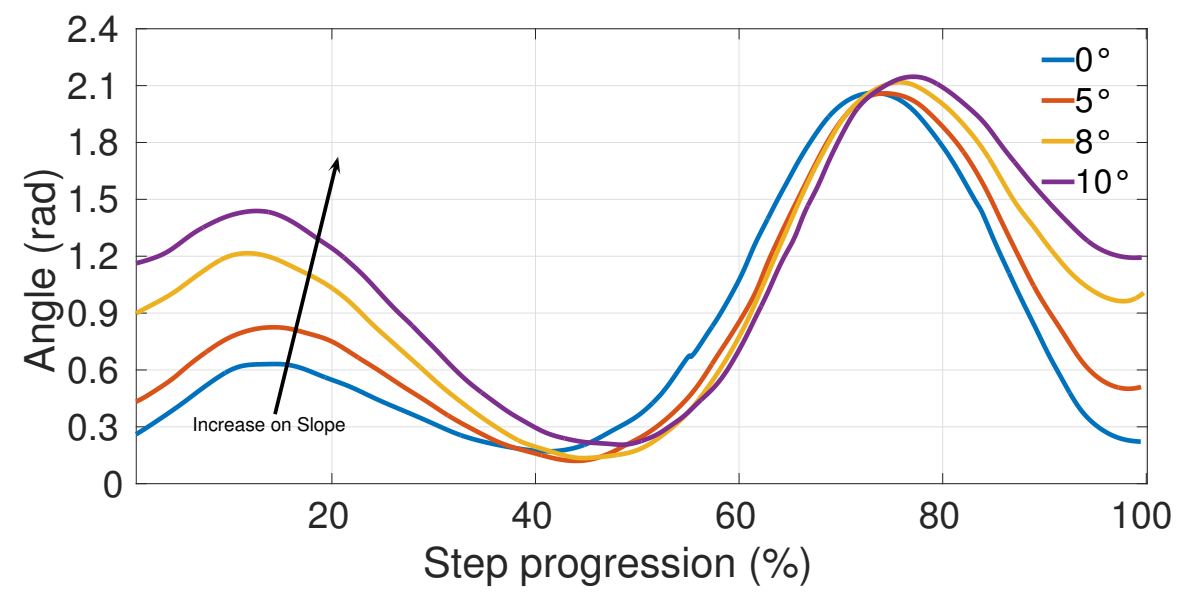

Figure 2.4: Knee trajectory for $0^{\circ}, 5^{\circ}, 8^{\circ}$ and $10^{\circ}$ slope inclinations. The data is taken from McIntosh [16]. ${ }^{1}$

\footnotetext{
${ }^{1}$ Reprinted from Journal of Biomechanics, Vol 39, Issue 13, Andrew Stuart McIntosh, Karen T. Beatty, Leanne N. Dwan and Deborah R. Vickers, Gait dynamics on an inclined walkway, Pages No. 2491 - 2502, Copyright (2006), with permission from Elsevier
} 


\subsection{Strategies for Upslope Walking}

Ankle and knee (Fig. 2.3 and Fig. 2.4) shows that most of the variation on trajectories is present in the extremes of the trajectories, in other words, at the beginning of stance phase and at the final part of the swing phase. In the next subsections a brief discussion about the human strategies used during upslope walking will be used to motivate the blending of flat ground walking into upslope walking in the context of a transfemoral prosthesis.

\subsubsection{Human Strategy}

Although a proper analysis of upslope walking should involve the dynamics [16], focusing on the kinematic trends allows to develop high-level strategies to 'mimic' the behavior of humans during upslope walking independently of the dynamics differences between a human leg and the prosthetic leg. The first values of ankle and knee in the walking progression (Fig. 2.3 and Fig. 2.4) are imposed by the inclined surface, in other words, the geometry of the slope modifies the angular positions of the ankle and knee. As the slope increases there is an increase in knee flexion and ankle dorsiflexion. Those results are consistent with [16], which reports that knee flexion at heel strike increase from $7^{\circ}$ from $33^{\circ}$ with an increase of surface inclination angle from $0^{\circ}$ to $+10^{\circ}$. Recall that knee is the most sensitive joint with respect to the slope inclination [16].

The differences in ankle and knee upslope trajectories with respect to flat ground can be considered as a new initial condition into the system, it is observed that from this initial condition that the trajectories converge into the nominal flat ground trajectories approximately at $40 \%$ of the step progression and stays on the flat ground walking gait until approximately $80 \%$ of the step progression, where the trajectories suffer deviations again because of the geometry of the surface. 


\subsubsection{Controller Strategy}

A simple algorithm is proposed to blend flat ground into upslope walking trajectories. This algorithm is based on two simple actions: i) generate in real time a trajectory that starts from the condition imposed by the surface (red shaded region in Fig. 2.6) and ii) use low gain PD control that can offer terrain adaptation at the final part of the step (blue shaded region in Fig. 2.6). The low gain PD control can shape the angles of the joints at the final part of the step progression in order to let the prosthesis to acquire more knee flexion as the slope increases (Fig. 2.5).

The generation of trajectories requires to be calculated in real time and to meet several constraints such as smoothness and boundedness for physically admissible velocities and accelerations. Considering the constraints and the real time necessity of the solution, the formulation takes the form of a convex optimization problem. The convex optimization formulation will be discussed later and will be merged into the human-inspired walking formulation to generate a complete framework that yields flat ground and upslope walking gaits.

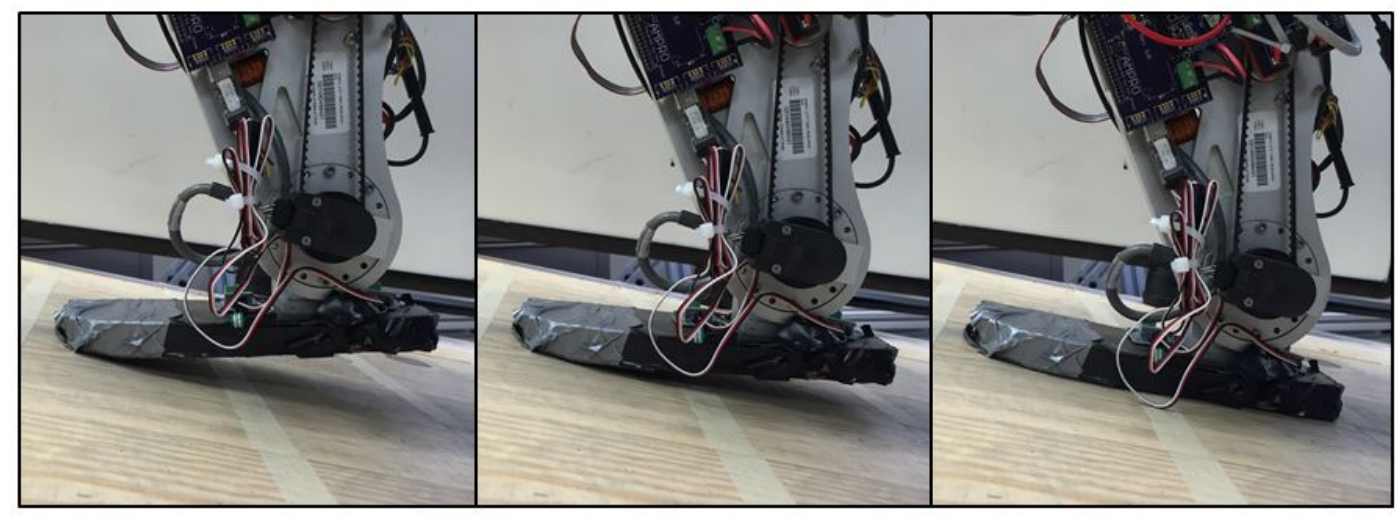

Figure 2.5: Low gain PD action used to enable terrain adaptation. 


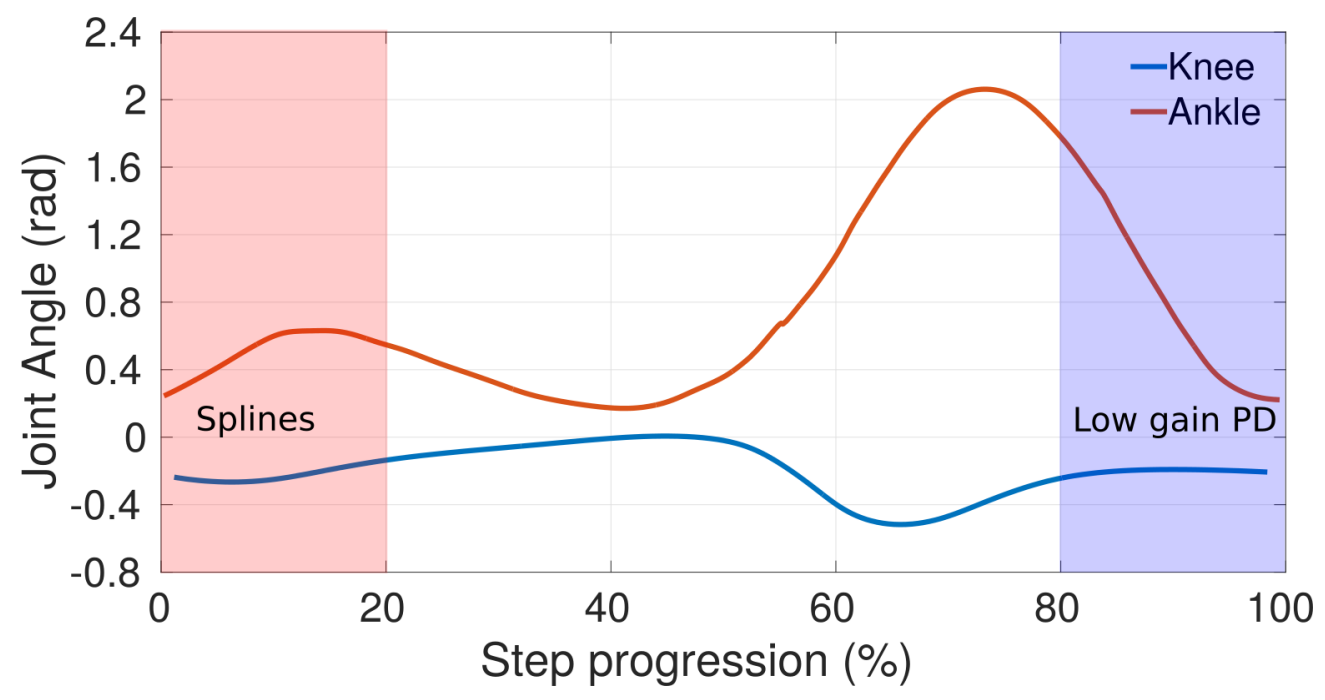

Figure 2.6: Typical ankle and knee flat ground trajectories. The red shaded region represents the action of the splines and the blue shaded region the action of the low PD control.

The low gain PD control on the ankle and knee act as a passive spring and damper with a fixed equilibrium, the parameters are chosen in this case to adapt to knee surfaces. The controller takes the following form:

$$
\tau_{i}=k p_{i}\left(\theta_{\text {actual }, i}-\theta_{\text {desired }, i}\right)+k d_{i}\left(\dot{\theta}_{\text {actual }, i}-\dot{\theta}_{\text {desired }, i}\right), \quad i \in\{\text { ankle }, k n e e\}
$$

where, $k_{i}, \theta_{i}$ and $b_{i}$ are the stiffness, equilibrium point and damping parameters describing the impedance. Note that low gain PD control is implemented in the last part of the step progression. When the splines are not used, the human-inspired control will drive the joints. 


\section{FLAT TERRAIN GAIT GENERATION}

The generation of upslope walking gaits starts from the construction of automatically stable and human-like flat ground trajectories using the human-inspired control approach. The HIC $[2,1,28]$ is a method based on the HZD approach $[27,26]$ that uses human-data as a reference for the construction of functions that represent the human walking through simple mathematical expressions. These functions are fully defined through a non-linear optimization problem that is used to guarantee stability while considering physical constraints of the device. By tracking these functions by an appropriate use of feedback control, the system becomes stable and performs human-like walking in bipedal robots and transfemoral prostheses.

\subsection{Bipedal Model}

A planar bipedal robotic model with 7 links (one torso, two thighs, two calves and two feet) which shares similar anthropomorphic values as the human using the prosthetic device is constructed to have a representation of the human in the generation of the walking gait. Analogously, the coordinates are chosen based on the

human subject (3.1) as $\theta=\left[\begin{array}{llllll}\theta_{s a} & \theta_{s k} & \theta_{s h} & \theta_{n s h} & \theta_{n s k} & \theta_{n s a}\end{array}\right]^{T} \in Q_{R}$, where $Q_{R}$ is the configuration space, a submanifold of $\mathbb{R}^{6}$.

The walking gait will be generated with the additional assumption of flat foot walking. It is considered that flat foot walking captures the essential movements of the walking gait [33] and is used as the testbed for implementation on more human-like walking representation such as multi-contact walking (for modeling multi-contact behavior see [30]). Additionally, during human walking, the impact with the ground plays a relevant event on the system that changes instantly the joint velocities [26]. In order to take in consideration this discrete event, the bipedal model is regarded 
as a hybrid system $[4,26]$ with continuous dynamics and a discrete event.

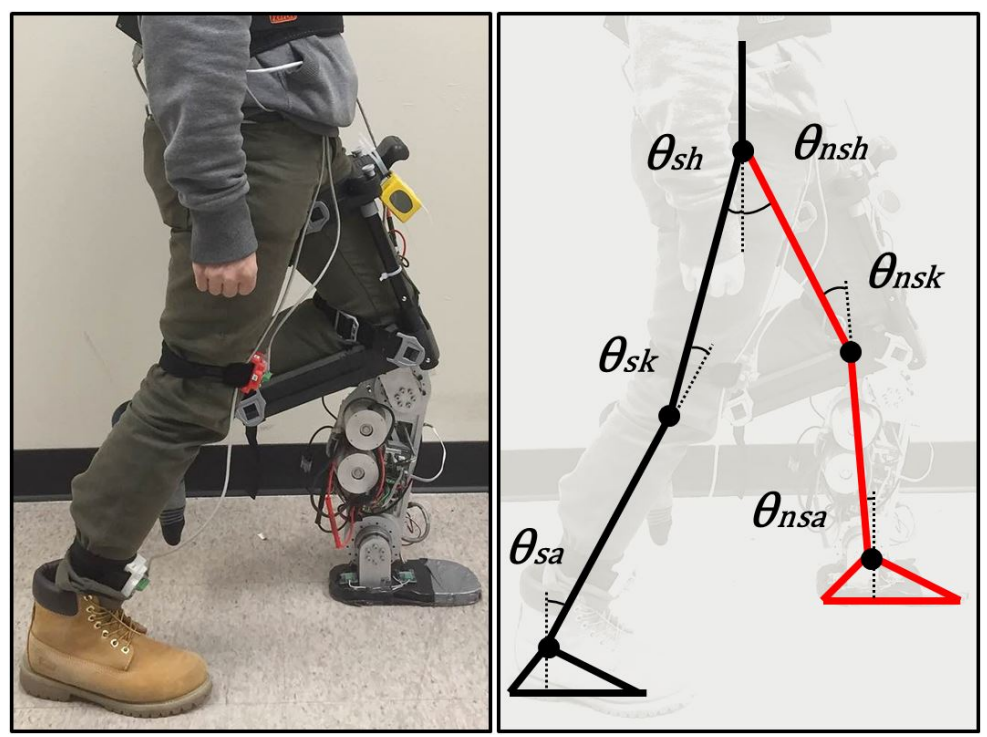

Figure 3.1: Bipedal robotic model considering the same anthropomorphic dimensions as the human subject.

The equations of motion representing the continuous dynamics of the system can be expressed as:

$$
D(\theta) \ddot{\theta}+H(\theta, \dot{\theta})=B u
$$

where, $D(\theta) \in \mathbb{R}^{6}$ is the inertial matrix and $H(\theta, \dot{\theta})=C(\theta) \dot{\theta}+G(\theta), C(\theta)$ the Coriolis term and $G(\theta)$ the gravity vector. Since we are dealing with full actuation due to the flat foot assumption, the torque map is the identity matrix, $B=I_{6}$. The input to the system is given by the torques $u$. Expressing (3.1) in terms of state space variable 
$x=(\theta, \dot{\theta})^{T}$, we found an affine control system representation as shown below:

$$
\dot{x}=f(x)+g(x) u
$$

where,

$$
\begin{array}{r}
f(x)=\left[\begin{array}{c}
\dot{\theta} \\
-D^{-1}(\theta) H(\theta, \dot{\theta})
\end{array}\right] \\
g(x)=\left[\begin{array}{c}
0 \\
D^{-1}(\theta) B(\theta)
\end{array}\right]
\end{array}
$$

Definition 3.1.1. A hybrid control system is a tuple:

$$
\mathcal{H C}=(X, U, S, \Delta, f, g)
$$

where,

- $X$ is a smooth submanifold of $\mathbb{R}^{6}$.

- $U \subseteq \mathbb{R}^{6}$ is the space of admissible controls.

- $S \subsetneq X$ is the guard or switching surface.

- $\Delta: S \rightarrow X$ is the reset map.

- $(f, g)$ is a control system s.t $\dot{x}=f(x)+g(x) u$.

To discriminate between single support phase and double support phase in the walking cycle, the unilateral constraint $h(\theta)$ is used. This constraint is the distance of the swing foot with respect to the ground, while this constraint is positive $(h(\theta)>0)$, it ensures that the swing foot is above the ground, i.e the system is in single support 
phase. An impact occurs when the unilateral constraint becomes zero $(h(\theta)=0)$ and the velocity of impact is less or equal than zero $(\dot{h}(\theta) \leq 0)$. The single support phase can be expressed as:

$$
X=\left\{(\theta, \dot{\theta}) \in T Q_{R}: h(\theta) \geq 0\right\}
$$

where, $T Q_{R}$ is the tangent bundle of the system. The guard is defined as the boundary of the domain with the unilateral constraint decreasing, in other words, when the leg is impacting the ground with certain velocity. The guard can be expressed as:

$$
S=\left\{(\theta, \dot{\theta}) \in T Q_{R}: h(\theta)=0, \frac{d h(\theta)}{d t} \leq 0\right\}
$$

To model the discrete event (impact), it is assumed that the impacts are plastic with impulsive forces acting on the system leg at the moment of contact. It is required to consider the extended coordinates that include the position of the support foot given by $\theta_{e}=\left(p_{x}, p_{z}, \theta\right)^{T} \in \mathbb{R}^{2} \times Q_{R}$. Assume that the position of the swing foot end is defined as $\Gamma\left(\theta_{e}\right)$ and its Jacobian is given by $J_{\Gamma}\left(\theta_{e}\right)$. After an impact occurs the velocity of the joints suffers an instantaneous change in velocity, described by the impact map:

$$
\dot{\theta}_{e}^{+}=P\left(\theta_{e}\right) \dot{\theta}_{e}^{-}=\left(I-D^{-1}\left(\theta_{e}\right) J_{\Gamma}^{T}\left(\theta_{e}\right)\left(J_{\Gamma}\left(\theta_{e}\right) D^{-1}\left(\theta_{e}\right) J_{\Gamma}^{T}\left(\theta_{e}\right)\right)^{-1} J_{\Gamma}\left(\theta_{e}\right)\right) \dot{\theta}_{e}^{-}
$$

Note that the joint positions does not change after the impact [26].

Whenever impacts occur the legs must be swapped, i.e, the support leg must be relabeled as the swing leg and the swing leg must be relabeled as the support leg. This is possible to achieve by the use of a coordinate transformation $\Re$. This transformation 
is included in the reset map $\Delta$.

$$
\Delta: S \rightarrow X, \quad \Delta(\theta, \dot{\theta})=\left[\begin{array}{ll}
\Re & 0 \\
0 & \Re
\end{array}\right]\left[\begin{array}{c}
\theta \\
P(\theta) \dot{\theta}
\end{array}\right]
$$

\subsection{Human-Inspired Control (HIC)}

With the appropriate model of the bipedal robotic system correlated with the human user, the next step towards the control design is the the selection of control objectives. The idea the tracking of canonical walking functions that capture the behavior of human walking, they are canonical in the sense that they are intrinsic in human walking. By tracking the canonical walking functions a robotic model can exhibit the same behavior as human walking despite the difference in dynamics $[1,20]$.

\subsubsection{Canonical Walking Functions (CWF)}

The canonical walking functions are inherent to human walking, in the sense that different subjects present similar canonical walking functions. This is supported by the experiments conducted by Ames [1]. Considering the 7-link bipedal robot with the flat foot assumption a total of 6 canonical walking functions are required (Fig. 3.2): $\delta p_{h i p}$ the linearized hip position, $\theta_{s k}$ the supporting knee, $\theta_{n s k}$ the nonsupporting knee, $\delta m_{n s l}$ the non-supporting slope, $\theta_{\text {torso }}$ the torso angle and $\theta_{n s f}$ the non-supporting foot. 

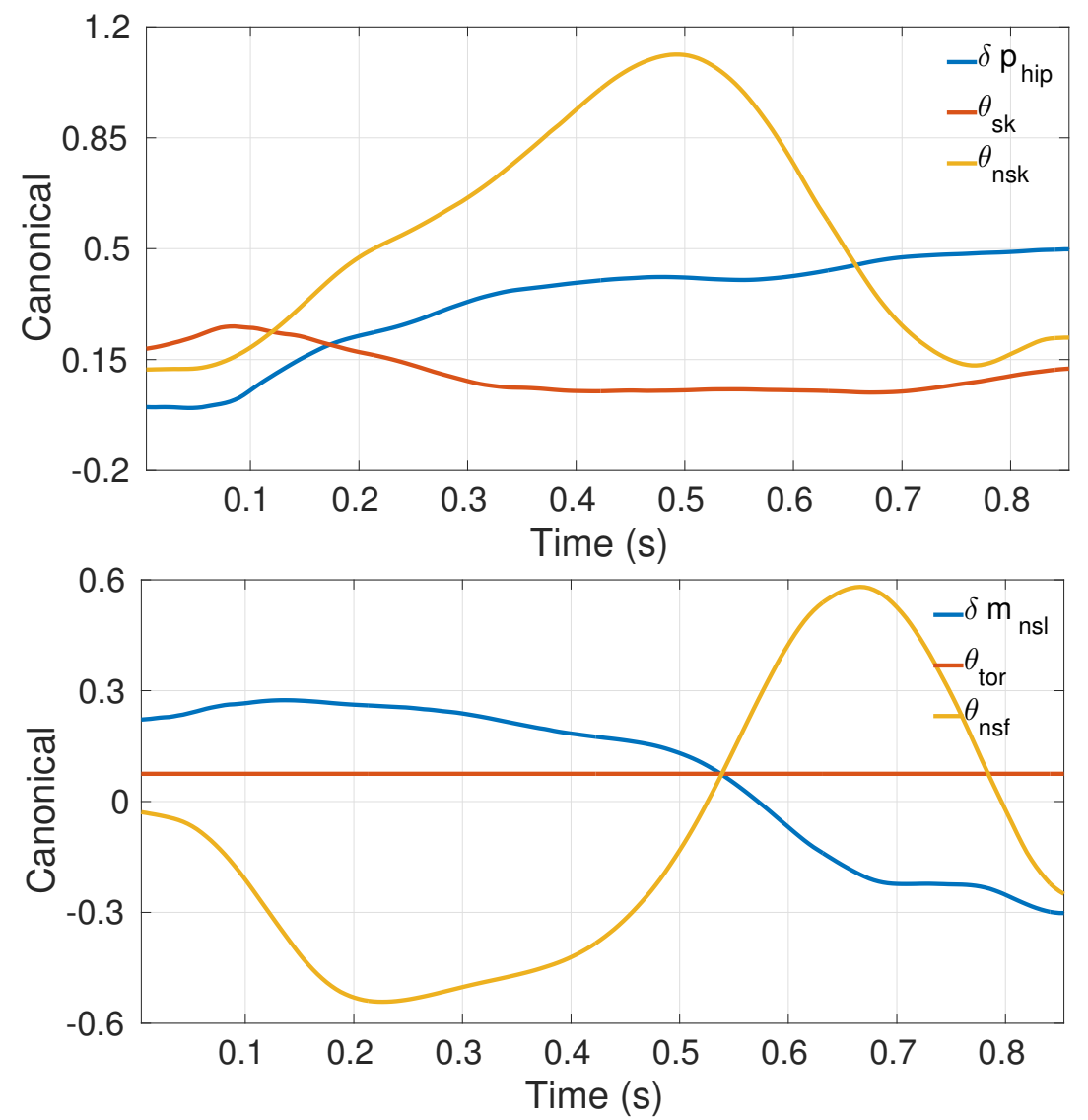

Figure 3.2: Human data collected though IMUs represented as canonical walking functions. They are conformed by the hip position $(\mathrm{m})$ and the joints relations $(\mathrm{rad})$.

The canonical walking functions have two different types of outputs, one of them corresponds to the linearized velocity of the hip $\left(y_{1}^{d}(\theta, \dot{\theta})\right)$ and the remaining can be modeled as the solution of a second order system $\left(y_{2}^{d}(\theta, \dot{\theta})\right)$.

$$
\begin{array}{r}
y_{1}^{d}(\theta, \dot{\theta})=\delta \dot{p}_{h i p}(\theta, \dot{\theta}) \\
y_{2}^{d}(\theta, \dot{\theta})=\left[\theta_{s k}, \theta_{n s k}, \delta m_{n s l}, \theta_{t o r}, \theta_{n s f}\right]^{T}
\end{array}
$$


In general, each output $y_{d}(t, \alpha)$ can be described as functions dependent on a set of parameters $\alpha$. Note that $v_{\text {hip }}$ is considered to be a member of $\alpha$.

$$
\begin{array}{r}
y_{d}^{1}(t)=v_{\text {hip }} t \\
y_{d, i}^{2}\left(t, \alpha^{i}\right)=e^{-\alpha_{4}^{i} t}\left(\alpha_{1}^{i} \cos \left(\alpha_{2}^{i} t\right)+\alpha_{3}^{i} \sin \left(\alpha_{2}^{i} t\right)\right)+\alpha_{5}^{i}
\end{array}
$$

where, $i \in\left\{\theta_{s k}, \theta_{s k}, \theta_{n s k}, \delta m_{n s l}, \theta_{\text {torso }}, \theta_{n s f}\right\}$.

\subsubsection{Human-Inspired Outputs (HIO)}

The human inspired outputs are the tracking errors between the actual and desired canonical walking functions. By driving them to zero $(y(\theta, \dot{\theta}, \alpha) \rightarrow 0)$, the robotic model will perform a human-like walking gait.

$$
y(\theta, \dot{\theta}, \alpha)=\left[\begin{array}{c}
y_{1}(\theta, \dot{\theta}) \\
y_{2}(\theta, \alpha)
\end{array}\right]=\left[\begin{array}{c}
y_{1}^{a}(\theta, \dot{\theta})-v_{\text {hip }} \\
y_{2}^{a}(\theta)-y_{2}^{d}(\rho(\theta), \alpha)
\end{array}\right]
$$

Note that $y_{1}(\theta, \dot{\theta})$ is a relative degree one output and $y_{2}(\theta, \alpha)$ corresponds to the relative degree two outputs [19]. $\rho(\theta)$ is used as a parameterization of time based on the observation that the hip position is regarded to increase linearly on time [1]. The parameterization is given as:

$$
\rho(\theta)=\frac{\delta_{p_{h i p}}(\theta)-\delta_{p_{h i p}}^{+}}{v_{h i p}}
$$

where, $\delta_{p_{h i p}}^{+}(\theta)$ is the position of the hip at the beginning of the step, designed in the human-inspired optimization, described in the next section and $\delta_{p_{\text {hip }}}(\theta)$ is the actual (linearized) position of the hip. The importance of the parameterization of time is that using $\rho(\theta)$ as a phase variable the user have control over the progression of 
the step, independent of time. The interaction between the user and the prosthesis makes it specially well suited for transfemoral prosthesis. The calculation of the phase variable $\rho(\theta)$ depends on which leg is supporting the user's weight. If the user is standing on the prosthesis leg, then the encoders are used to calculate $\rho$, otherwise, the IMUs attached to the healthy leg are used to estimate through a EKF the actual hip position [33].

\subsubsection{Control Design}

Considering the human-inspired outputs defined in (3.14) the idea is to drive these outputs to zero. Applying a feedback linearization control [19] to the outputs, the resulting control law is:

$$
\left[\begin{array}{c}
\dot{y_{1}} \\
\ddot{y_{2}}
\end{array}\right]=\underbrace{\left[\begin{array}{c}
L_{f y_{1}}(\theta, \dot{\theta}) \\
L_{f y_{2}}^{2}(\theta, \dot{\theta}, \alpha)
\end{array}\right]}_{L_{f}(\theta, \dot{\theta}, \alpha)}+\underbrace{\left[\begin{array}{c}
L_{g y_{1}}(\theta, \dot{\theta}) \\
L_{g} L_{f y_{2}}(\theta, \dot{\theta}, \alpha)
\end{array}\right]}_{\mathcal{A}(\theta, \dot{\theta}, \alpha)} u
$$

where, $L_{f}(\theta, \dot{\theta}, \alpha)$ represents the lie derivatives and $\mathcal{A}(\theta, \dot{\theta}, \alpha)$ is the decoupling matrix, which is invertible due to the selection of the outputs $[32,1]$.

Choosing a control a control input $u \in \mathcal{U}$ in the form $u=\mathcal{A}^{-1}(\theta, \dot{\theta}, \alpha)\left(-L_{f}(\theta, \dot{\theta}, \alpha)+\right.$ $\mu)$, the equation (3.14) becomes:

$$
\left[\begin{array}{c}
\dot{y_{1}} \\
\ddot{y_{2}}
\end{array}\right]=\mu
$$

Note that for the bipedal model, exact information about the dynamics can be obtained, it allows to perform a simulation of the system and observe the resulting walking gait. However, for implementation purposes the dynamics of the user are unknown, thus the implementation of the controller needs to be model independent 
such as PD control or Model Independent QP (MIQP) [33].

To drive the outputs to zero the form that $\mu$ take can be designed as:

$$
\mu=\left[\begin{array}{c}
-2 \epsilon y_{1} \\
-2 \epsilon L_{f y_{2}}-\epsilon^{2} y_{2}
\end{array}\right]
$$

\subsubsection{Partial Hybrid Zero Dynamics}

When the tracking outputs are driven to zero, the control law is expected to render the zero dynamics surface:

$$
Z_{\alpha}=\left\{(\theta, \dot{\theta}) \in T Q_{R}: y_{1}(\theta, \dot{\theta})=0, y_{2}(\theta, \alpha)=0, L_{f} y_{2}(\theta, \dot{\theta}, \alpha)=0\right\}
$$

This surface describes an invariant set for the continuous dynamics, once the tracking objectives are driven to zero, then the outputs will stay at zero for posterior times, however it is desired to guarantee that the outputs are impact invariant as well. The dependence of the surface with the parameter $\alpha$ allows the search of canonical walking functions that can render the surface hybrid invariant [27]. Additionally, a less restrictive surface that allows changes in the hip velocity $\left(y_{1}^{d}=v_{\text {hip }}\right)$ after the impact, might help to compensate to impulsive effect of impacts and the modeling errors, this surface is the Partial Hybrid Zero Dynamics (PHZD).

$$
P Z_{\alpha}=\left\{(\theta, \dot{\theta}) \in T Q_{R}: y_{2}(\theta, \alpha)=0, L_{f} y_{2}(\theta, \dot{\theta}, \alpha)=0, h(\theta)>0\right\}
$$

An impact on the system affects the joint velocities (3.8), this can deviate the system evolution away from the PHZD. In order to constrain the system to be invariant under impacts it is necessary to ensure that when the system is in the switching 
surface $S$ evolving in the PHZD surface, an impact $\Delta$ does not move the system away from the PHZD surface $P Z_{\alpha}$. The PZHD constraint can be stated as:

$$
\Delta\left(S \cap P Z_{\alpha}\right)=P Z_{\alpha}
$$

Therefore, the goal is to select the parameters $\alpha$ such that the system renders the PHZD invariant under impacts $[27,1]$. This can be enforced as a constraint in the human-inspired optimization.

\subsection{Human-Inspired Optimization (HIO)}

The PHZD constraint generates human-like walking gaits that are provably stable $[1,28,30]$. More constraints must be imposed considering the limitations of the prosthesis, such as torque, position and velocity limits. All the requirements of the walking gait must be specified in the optimization problem along with the physical limitations of the device itself.

$$
\begin{array}{r}
\alpha^{*}=\underset{\alpha \in \mathbb{R}^{26}}{\operatorname{argmin}} \operatorname{Cost}_{H D}(\alpha) \\
\Delta\left(S \cap P Z_{\alpha}\right)=P Z_{\alpha}
\end{array}
$$

(Physical constraints)

The cost function $\operatorname{Cost}_{H D}(\alpha)$ is the square weighted square error between human data and the CWF, as shown in the expression below. Complete details of the optimization can be found in [1].

$$
\operatorname{Cost}_{H D}(\alpha)=\sum_{k=1}^{K}\left[\left(\beta_{y_{1}}\left(y_{1}\left(t[k], v_{h i p}\right)-y_{1}^{H}(k)\right)\right)^{2}+\sum_{j=1}^{5} \beta_{y_{2, j}}\left(y_{2, j}(t[k], \alpha)-y_{2, j}^{H}(k)\right)^{2}\right]
$$


where, $K$ is the total number of points taken during the motion capture, $y_{1}^{H}(k)$ is the vector of values corresponding to the human hip position at index $k$. The relative degree two human data is labeled as $y_{2, j}^{H}(k)$ being the index $j$ the selector of one of the five relative degree two functions. The weights $\beta$ are defined as the difference between the maximum and the minimum value of the output data.

$$
\begin{array}{r}
\beta_{y_{1}}=1 /\left(\max _{k}\left(y_{1}^{H}(k)\right)-\min _{k}\left(y_{1}^{H}(k)\right)\right) \\
\beta_{y_{2, j}}=1 /\left(\max \left(y_{2, j}^{H}(k)\right)-\min \left(y_{2, j}^{H}(k)\right)\right), \quad j \in[1,5]
\end{array}
$$

A Poincare analysis is carried out to guarantee stability ensuring that the eigenvalues are less than $1[25,18,1]$.

The strength of the human-inspired optimization is the automatic generation of stable and human-like walking gaits that requires no parameter tuning or calibration. Additionally, it allows to consider system constraints so the device can evolve in a feasible manner inside the designed bounds [33].

\subsection{Motion Capture System}

To acquire human data a IMU-based motion capture system was used. The IMUs consisted in seven MPU-9150 devices (tri-axis gyroscope and tri-axis accelerometer), they were placed in fixed places in the feet, calves, thighs and torso. A kinematic model is constructed in the sagital plane starting from the hip, with the assumption that the hip acceleration is negligible. During the data recording procedure, an EKF is utilized to estimate the joint trajectories, which will be used as the references for the prosthetic gait design.

The human data captured by the IMU-based motion system is utilized on the humaninspired optimization (Fig. 3.2), and particularly Fig. 3.3 shows the ankle and knee 
joints.

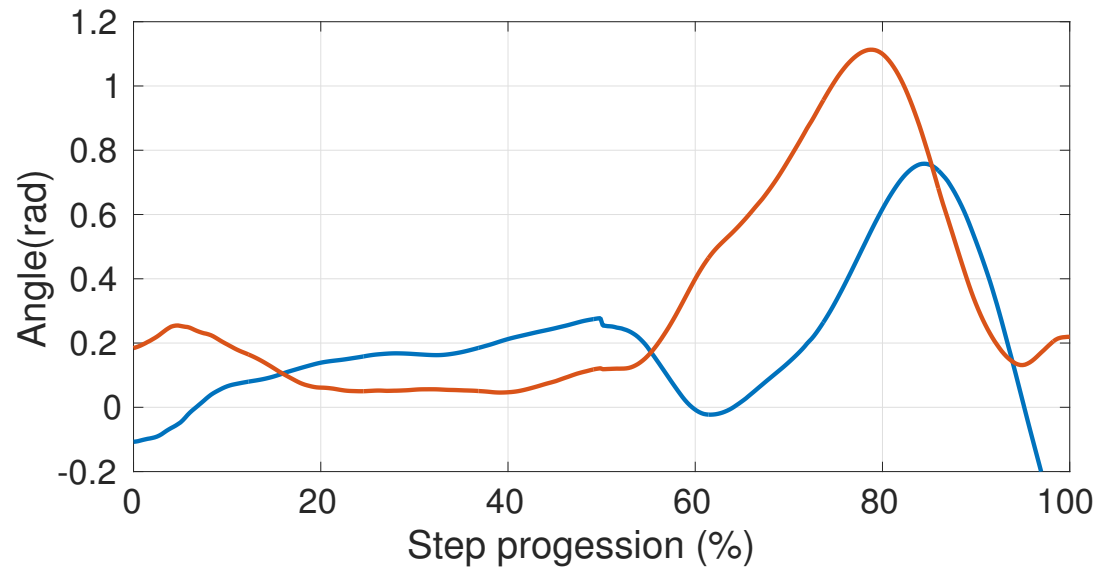

Figure 3.3: Trajectories captured by the IMU-based motion system for ankle and knee.

Solving the human-inspired optimization gives as a result the parameters $\alpha$ required to fully define the canonical walking functions. The ankle and knee trajectories generated for AMPRO II are shown in Fig. 3.4. Note that the trajectories of the knee present differences with respect to normal human walking, it is because of the flat foot behavior requested to the subject. 


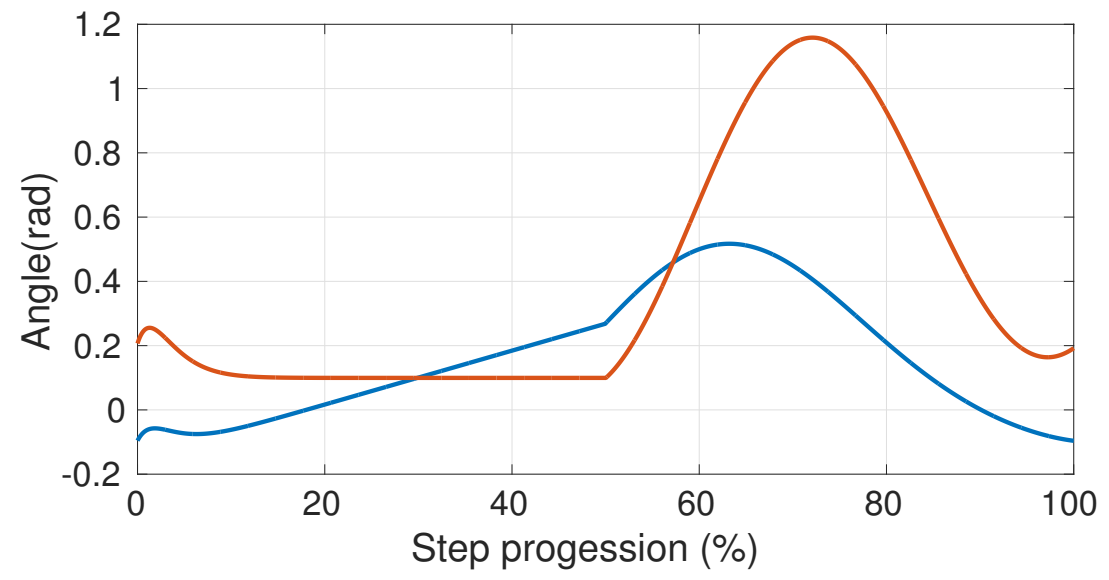

Figure 3.4: Trajectories generated by the human-inspired optimization for ankle and knee.

The phase portrait plot shown in Fig. 3.5 exhibits that the trajectories evolve into a continuous curve and then they are affected by a discrete impact (the discontinuity). Note that the discrete impact keeps the angle values but changes the joint velocities instantaneously (3.8).

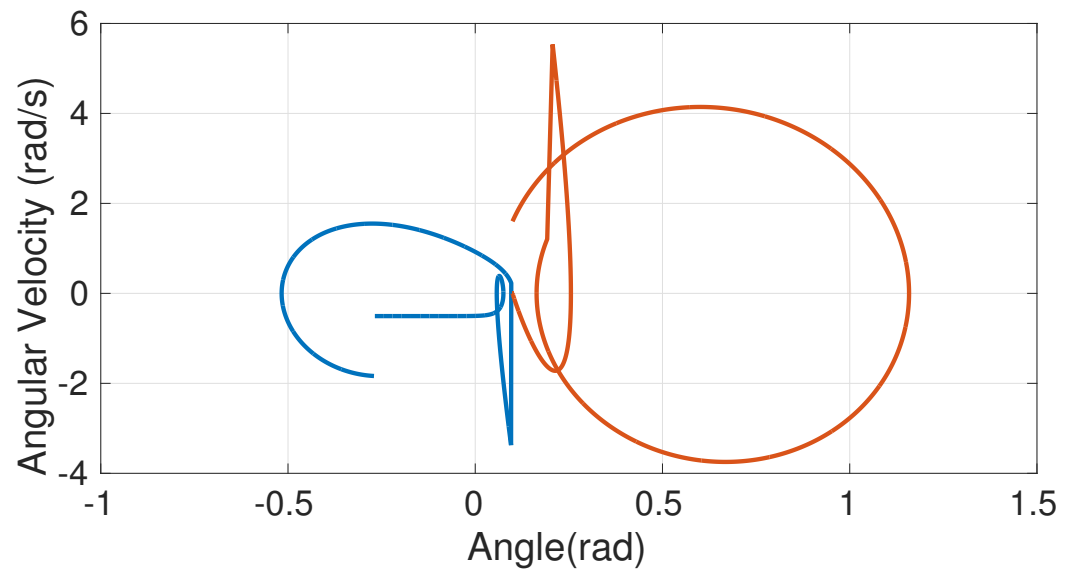

Figure 3.5: Ankle and knee phase portrait, indicating a continuous evolution of the system and a discrete change in velocity at impact. 


\subsection{Simulation of the Flat Ground Gait}

The simulation performed using feedback linearization generated an stable and human-like walking gait, represented in the walking tiles in Fig. 3.7.
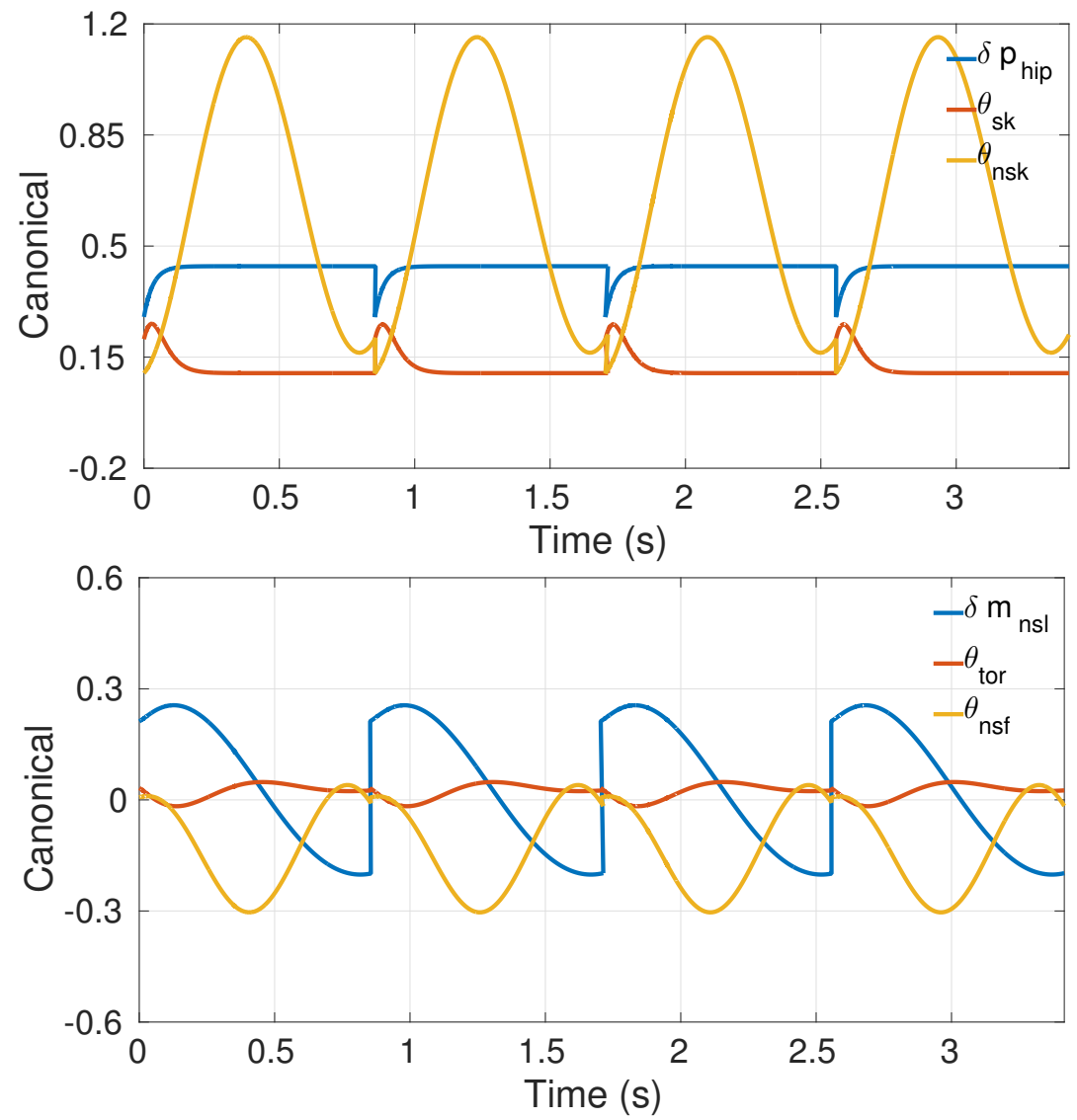

Figure 3.6: Canonical walking functions obtained in the simulation.

The canonical walking functions generated for this particular walking gait are shown in Fig. 3.6 for 4 steps. Note that hip velocity suffers a change after the impact but it converges to its desired value eventually. 


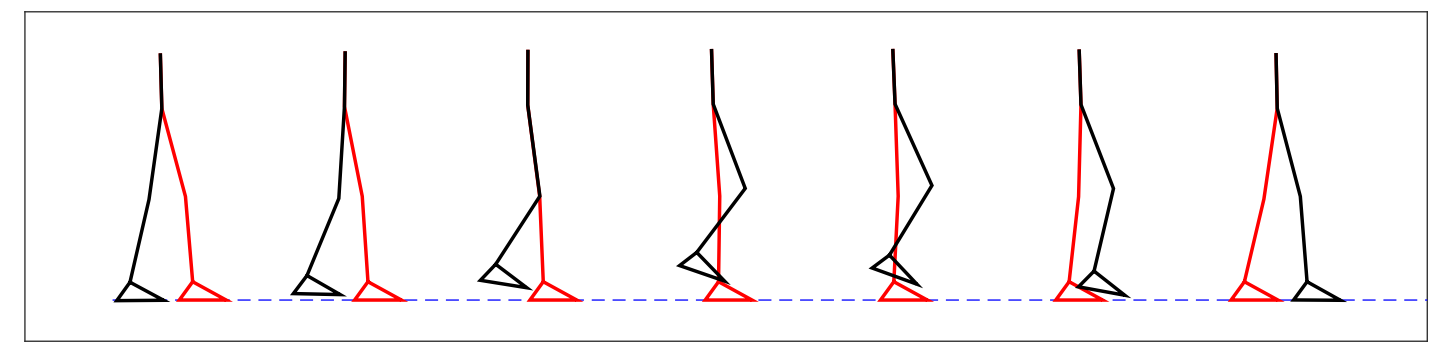

Figure 3.7: Gait tile simulation using feedback linearization control.

\subsection{Gait Generation for Non-Flat Terrain}

The human-inspired control can be used to generate walking gaits for diverse terrains. This section will summarize the application of this framework for rough terrain and for stair ascending. Additionally it presents a discussion about the limitations of the HIC framework and possible options to address adaptability for transfemoral prostheses. The summary of the additional methods is rather informal but references are provided for more details.

\subsubsection{Extended Canonical Walking Functions (ECWF) for Rough Terrain}

Rough terrain imposes a challenge on the generation of human-inspired walking gaits due to the unknown and unpredictable terrain profile, Shishir [9] proposed that one solution to handle this problem is the modification of the CWF into the extended canonical walking functions $(\mathrm{ECWF})$. The ECWF are constructed by the addition of more parameters that can be calculated in real time, depending on the initial conditions imposed by the surface.

$y_{2}^{e}(t, \alpha)=e^{-\alpha_{4} t}\left(\alpha_{1} \cos \left(\alpha_{2} t\right)+\alpha_{3} \sin \left(\alpha_{2} t\right)\right)+\alpha_{5} \cos \left(\alpha_{6} t\right)+\frac{2 \alpha_{5} \alpha_{4} \alpha_{2}}{\alpha_{2}^{2}+\alpha_{4}^{2}-\alpha_{6}^{2}} \sin \left(\alpha_{6} t\right)+\alpha_{7}$ 
Choosing the linear terms $\alpha_{v}=\left[\alpha_{1}, \alpha_{3}, \alpha_{5}, \alpha_{7}\right]$ we can construct a linear matrix equation by keeping $\alpha_{2}, \alpha_{4}, \alpha_{6}$ constant and solving the equation in order to accommodate the condition imposed by the surface [9]. In this manner, it allows freedom in the terrain profile by calculating a new $\alpha_{v}$ at every step.

\subsubsection{Upstairs Walking}

The human-inspired optimization is highly dependent on the human-data and the terrain profile, using data for upstairs walking it would be possible to solve the HIO given an appropriate redefinition of the guard $S$ (3.7). In particular, the guard must define the terrain profile exactly in order to account for the impacts that happen during walking. Huihua et al. [29] generated a walking gait based on the humaninspired optimization given a priori knowledge of the stairs profile, the walking gait was implemented successfully into the prosthetic device AMPRO I.

\subsubsection{Limitations}

Note that in order to provide adaptability to new terrains it is important to generate joint trajectories that resemble the trajectories followed by healthy human subjects, for instance, while ECWF works for rough terrain, it cannot provide a specific desired behavior during upslope walking. Furthermore, if we are to use the human-inspired optimization for the generation of walking gaits at each new terrain, this problem could be unfeasible, as currently there is no solution on real time for this optimization. Additionally, in practical implementation with the prosthesis it is not possible to acquire information of the terrain profile.

In consequence, terrain adaptability requirements are twofold: i) Generation of trajectories with specific desired behaviors and ii) online solutions for the generation of new trajectories at every step. Regarding upslope walking, the generation of an algorithm that holds the two conditions for terrain adaptability suggests a convex 
optimization formulation. The reason for choosing a convex optimization problem is because it is fast to solve and can be subject to constraints and objectives that can be formulated to mimic the behavior of upslope walking.

The next chapter will introduce the convex optimization problem that is aimed to the blending of the flat ground trajectories already generated into upslope walking by mimicking the qualitative strategies found in Chapter 2. 


\section{OPTIMIZATION-BASED SPLINE GENERATION FOR UPSLOPE WALKING}

This chapter is motivated by the strategies realized by humans during upslope walking. A convex optimization based cubic spline problem is proposed due to its flexibility to be shaped into a desired behavior or trajectories and for the possibility to introduce constraints into the system that can be solved in real-time $[10,3]$. Furthermore, an analytical solution is proposed that can be solved easily with most linear algebra packages in the microcomputer used in the prosthesis.

\subsection{Problem Formulation}

The generation of a trajectory $S$ connecting a point (say, a point from trajectory $C 1$ ) to a nominal trajectory (say, trajectory $C 2$ ) as can be seen in Fig. 4.1 is the main goal of this chapter. In particular, a point in $C 1$ can be considered as the initial condition imposed by the inclined surface and $C 2$ the flat ground walking trajectories.

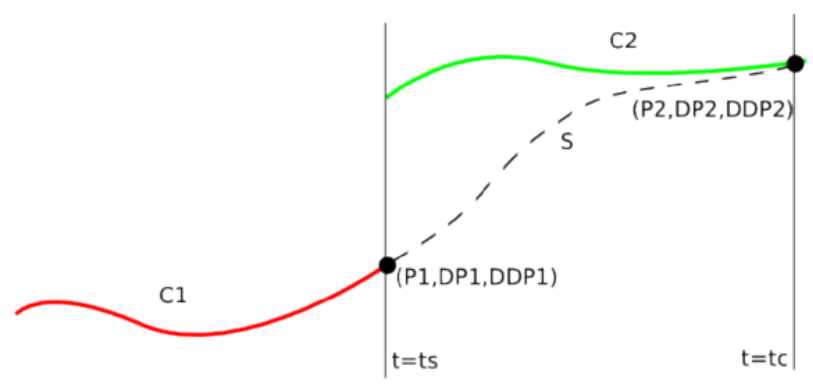

Figure 4.1: Two disconnected trajectories $C 1$ and $C 2$ can be connected trough a trajectory $S$, starting from time $t_{s}$ and finishing on $t_{c}$ 
Additionally, some important constraints must be met such as continuity in the connection points with $C 1$ and $C 2$ and smoothness on the entire trajectory. A discontinuity in position would impose high velocities that can affect the performance of the walking gait, analogously a dicontinuity in velocities can impose large accelerations.

A well-known solution to this problem is using a single cubic spline to connect the desired points. Cubic splines have four tunable parameters which can take care of continuity of position and velocity. Unfortunately, using a single spline lacks the capability to provide smooth accelerations at the extreme points, also this single cubic spline cannot be shaped to follow a desired qualitative behavior (by fitting a trajectory). This solution can be improved using several intermediate points (waypoints) connecting cubic splines between the origin and destination points. This technique is more advantageous as continuity, smoothness and obstacle avoidance could be achieved by picking appropriate spline parameters [10]. The usage of waypoints and a set of splines (Fig. 4.2) can be exploited to define a cost function that allows to shape the behavior of the set of cubic splines by minimizing the distance to some reference curve. 


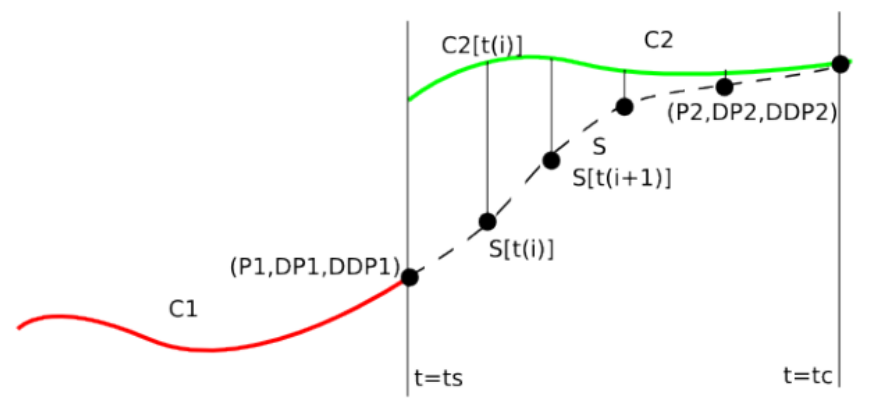

Figure 4.2: Connecting two different trajectories using a set of splines joined by specific waypoints

To guarantee that position, velocity and acceleration are smooth, it is necessary to check the waypoints. Each waypoint (except the extreme points) connects two splines. Both connected splines should have the same values in position, velocity and acceleration at each waypoint (Fig. 4.3).

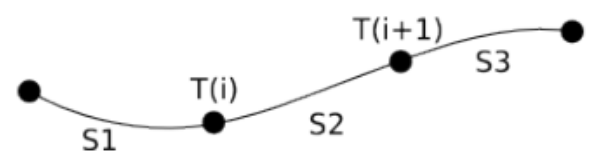

Figure 4.3: Cubic splines connected through waypoints

Assuming that the set of $\mathrm{N}$ splines $S_{1}, S_{2}, \ldots, S_{N}$ is represented by $S$, which is defined appropriately such that for a corresponding time $t$ it takes the value of the corresponding spline $S_{k}(t), k \in[1, N]$. Particularly at the waypoints the time is defined by $T(i)$ for $i \in[1, N]$. The mathematical representation for smoothness in 
position, velocity and acceleration can be written as:

$$
\begin{aligned}
& S_{1}(T(i))=S_{2}(T(i)) \\
& \dot{S}_{1}(T(i))=\dot{S}_{2}(T(i)) \\
& \ddot{S}_{1}(T(i))=\ddot{S}_{2}(T(i))
\end{aligned}
$$

Since the objective is to generate a convergent trajectory that follows a desired behavior, it is necessary to have a cost function based on the distance between a reference trajectory and the generated splines, Fig. 4.2 shows the distance between each waypoint to the desired trajectory. These distances will be used for the construction of the cost function, which is minimized through a least square formulation:

$$
\begin{gathered}
\min \sum_{i}\|S(T(i))-C 2(T(i))\| \\
\text { s.t. } \quad(4.1)
\end{gathered}
$$

(Smoothness constraints)

In order to describe the smoothness conditions, assume that each cubic spline has the following form:

$$
S_{i}=a_{0}^{i}+a_{1}^{i}\left(t-t_{c}\right)+a_{2}^{i}\left(t-t_{c}\right)^{2}+a_{3}^{i}\left(t-t_{c}\right)^{3}
$$

where, $\lambda_{i}=\left\{a_{0}^{i}, a_{1}^{i}, a_{2}^{i}, a_{3}^{i}\right\}$ is the set of parameters that defines the spline, note that $t_{c}$ is the convergence time (Fig. 4.1) and $t_{s}$ is the switching time. To include conditions of smoothness for velocity and acceleration, it is necessary to calculate 
the derivatives and double derivatives of the cubic spline.

$$
\begin{array}{r}
\dot{S}_{i}=a_{1}^{i}+2 a_{2}^{i}\left(t-t_{c}\right)+3 a_{3}^{i}\left(t-t_{c}\right)^{2} \\
\ddot{S}_{i}=2 a_{2}^{i}+6 a_{3}^{i}\left(t-t_{c}\right)
\end{array}
$$

To make the splines $S_{i}$ and $S_{i+1}$ to be continuous and smooth in terms of position, velocity and acceleration, its values at the waypoints should be equal. In the case of position, the conditions of smoothness are expressed as:

$$
\begin{array}{r}
S_{i}\left(t_{i}\right)=a_{0}^{i}+a_{1}^{i}\left(\Delta_{t_{i}}\right)+a_{2}^{i}\left(\Delta_{t_{i}}\right)^{2}+a_{3}^{i}\left(\Delta_{t_{i}}\right)^{3} \\
S_{i+1}\left(t_{i}\right)=a_{0}^{i+1}+a_{1}^{i+1}\left(\Delta_{t_{i}}\right)+a_{2}^{i+1}\left(\Delta_{t_{i}}\right)^{2}+a_{3}^{i+1}\left(\Delta_{t_{i}}\right)^{3} \\
S_{i}\left(t_{i}\right)-S_{i+1}\left(t_{i}\right)=0
\end{array}
$$

Considering smoothness in velocity, the conditions are expressed as:

$$
\begin{array}{r}
\dot{S}_{i}\left(t_{i}\right)=a_{1}^{i}+2 a_{2}^{i}\left(\Delta_{t_{i}}\right)+3 a_{3}^{i}\left(\Delta_{t_{i}}\right)^{2} \\
\dot{S}_{i+1}\left(t_{i}\right)=a_{1}^{i+1}+2 a_{2}^{i+1}\left(\Delta_{t_{i}}\right)+3 a_{3}^{i+1}\left(\Delta_{t_{i}}\right)^{2} \\
\dot{S}_{i}\left(t_{i}\right)-\dot{S}_{i+1}\left(t_{i}\right)=0
\end{array}
$$

Analogously for the smoothness conditions in acceleration:

$$
\begin{array}{r}
\ddot{S}_{i}\left(t_{i}\right)=2 a_{2}^{i}+6 a_{3}^{i}\left(\Delta_{t_{i}}\right) \\
\ddot{S}_{i+1}\left(t_{i}\right)=2 a_{2}^{i+1}+6 a_{3}^{i+1}\left(\Delta_{t_{i}}\right) \\
\ddot{S}_{i}\left(t_{i}\right)-\ddot{S}_{i+1}\left(t_{i}\right)=0
\end{array}
$$

These constraints can be written in matricial form. Consider a vector $\lambda$ storing 
all the parameters of the splines, a segment of the vector $\lambda_{i, i+1}$ is constructed in the following way:

$$
\lambda_{i, i+1}=\left[\begin{array}{llllllll}
a_{0}^{i} & a_{1}^{i} & a_{2}^{i} & a_{3}^{i} & a_{0}^{i+1} & a_{1}^{i+1} & a_{2}^{i+1} & a_{3}^{i+1}
\end{array}\right]^{T}
$$

Then, the constraints (4.8), (4.11) and (4.14) can be written as:

$$
\begin{array}{r}
{\left[\begin{array}{llllllll}
1 & \Delta_{t_{i}} & \Delta_{t_{i}}^{2} & \Delta_{t_{i}}^{3} & -1 & -\Delta_{t_{i}} & -\Delta_{t_{i}}^{2} & -\Delta_{t_{i}}^{3}
\end{array}\right] \lambda_{i, i+1}=0} \\
{\left[\begin{array}{lllllllll}
0 & 1 & 2 \Delta_{t_{i}} & 3 \Delta_{t_{i}}^{2} & 0 & -1 & -2 \Delta_{t_{i}} & -3 \Delta_{t_{i}}^{2}
\end{array}\right] \lambda_{i, i+1}=0} \\
{\left[\begin{array}{lllllllll}
0 & 0 & 2 & 6 \Delta_{t_{i}} & 0 & 0 & -2 & -6 \Delta_{t_{i}}
\end{array}\right] \lambda_{i, i+1}=0}
\end{array}
$$

The initial point continuity conditions $\left(P 1, D P 1, D^{2} P 1\right)$ are expressed using the first parameters of $\lambda$, using the following expression:

$$
\begin{gathered}
{\left[\begin{array}{cccccccc}
1 & \Delta_{t_{0}} & \Delta_{t_{0}}^{2} & \Delta_{t_{0}}^{3} & 0 & 0 & 0 & 0
\end{array}\right] \lambda_{1,2}=P 1} \\
{\left[\begin{array}{llllllll}
0 & 1 & 2 \Delta_{t_{0}} & 3 \Delta_{t_{0}}^{2} & 0 & 0 & 0 & 0
\end{array}\right] \lambda_{1,2}=D P 1} \\
{\left[\begin{array}{llllllll}
0 & 0 & 2 & 6 \Delta_{t_{0}} & 0 & 0 & 0 & 0
\end{array}\right] \lambda_{1,2}=D^{2} P 1}
\end{gathered}
$$

Analogous formulation for the final point in its position, velocity and acceleration values $P 2, D P 2, D^{2} P 2$ can be constructed.

$$
\begin{gathered}
{\left[\begin{array}{cccccccc}
0 & 0 & 0 & 0 & 1 & \Delta_{t_{N}} & \Delta_{t_{N}}^{2} & \Delta_{t_{N}}^{3}
\end{array}\right] \lambda_{N-1, N}=P 2} \\
{\left[\begin{array}{cccccccc}
0 & 0 & 0 & 0 & 0 & 1 & 2 \Delta_{t_{N}} & 3 \Delta_{t_{N}}^{2}
\end{array}\right] \lambda_{N-1, N}=D P 2} \\
{\left[\begin{array}{llllllll}
0 & 0 & 0 & 0 & 0 & 0 & 2 & 6 \Delta_{t_{N}}
\end{array}\right] \lambda_{N-1, N}=D^{2} P 2}
\end{gathered}
$$


All the constraints can be written in a single matrix, which is represented by the constraint matrix $C$.

$$
C \lambda=d
$$

where, $d$ is the value that the equation must take in order to meet the constraint conditions, which can be $P 1, D P 1, D^{2} P 1, P 2, D P 2, D^{2} P 2$ or zero depending on the waypoint and the condition being enforced. Recall that $\lambda$ is the collection of all the parameters, that is, $\lambda=\left[\begin{array}{lll}\lambda_{1} & \ldots & \lambda_{N}\end{array}\right]$

The cost function is constructed evaluating the splines at each waypoint:

$$
S_{i}\left(t_{i}\right)=\left[\begin{array}{cccc}
1 & \Delta_{t_{i}} & \Delta_{t_{i}}^{2} & \Delta_{t_{i}}^{3}
\end{array}\right] \lambda_{i}=P_{i} \lambda_{i}
$$

Then, all the values of the splines at the waypoints can be calculated in vector form, by the appropriate construction of the matrix $\mathrm{P}$, generating the following equation:

$$
S=P \lambda
$$

Finally, the minimization problem can be formulated as:

$$
\begin{aligned}
& \min \|P \lambda-Y\| \\
& \text { s.t } \quad C \lambda=d
\end{aligned}
$$

where, $Y$ is the desired set of points describing the reference trajectory, in particular for the thesis $Y=C 2$. Note that the problem can be solved in real time applications given its convex form. Moreover, an analytic solution can be obtained that can be 
implemented on the microcomputer of the prosthetic device AMPRO II.

\subsection{Analytical Solution}

Considering the problem expressed in the optimization:

$$
\begin{aligned}
& \min \|P \lambda-Y\| \\
& \text { s.t. } \quad C \lambda=d
\end{aligned}
$$

It is possible to rewrite the optimization problem as:

$$
\begin{array}{r}
\min \|P \lambda-Y\|=\min \quad \lambda^{T} P^{T} P \lambda-2 Y^{T} P \lambda+Y^{T} Y \\
\text { s.t. } \quad C \lambda=d
\end{array}
$$

Then, the lagrangian of the optimization problem can be expressed as:

$$
L=\lambda^{T} P^{T} P \lambda-2 Y^{T} P \lambda+Y^{T} Y+\nu^{T}(C \lambda-d)
$$

Using the KKT conditions for optimality:

$$
\begin{aligned}
2 P^{T} P \lambda-2 P^{T} Y+C^{T} \nu & =0 \\
C \lambda & =d
\end{aligned}
$$

It represents a matricial linear equation on $(\lambda, \nu)$ that can be expressed as:

$$
\left(\begin{array}{cc}
2 P^{T} P & C^{T} \\
C & 0
\end{array}\right)\left(\begin{array}{l}
\lambda \\
\nu
\end{array}\right)=\left(\begin{array}{c}
2 P^{T} Y \\
d
\end{array}\right)
$$

This equation can be solved for $\lambda$ and $\nu$ easily using Eigen Libraries in $\mathrm{C}++$, 
which can be implemented in real time and fits our requirements of implementation. An Eigen-based library has been created for the generation of a family of splines satisfying the conditions of smoothness and continuity. The public repository is located in: bitbucket.org/pvictorm6/splines_support

\subsection{Human-Inspired Walking with Cubic Splines}

The first connection between the set of cubic splines and the canonical walking functions is given by their parameterization in time. However, as mentioned earlier, a replacement of time is given by $\rho(\theta)$ in (3.15) which will be adopted by the splines as well. To fully define the domain of the splines, the boundary conditions must be explicitly stated, the splines will take action while $\rho(\theta) \in\left[t_{s}, t_{s}\right]$, otherwise, the canonical walking functions for flat ground walking will be used.

As soon as the prosthetic foot lands on a arbitrary surface, the values of its position, velocity and acceleration can be used for the boundary conditions of the initial point $\left(P 1, D P 1, D^{2} P 1\right)$. Considering that the objective is the connection of this point to its respective flat ground CWF, the final boundary conditions $(P 2$, $\left.D P 2, D^{2} P 2\right)$ are defined in terms of the desired values of the relative degree two canonical walking functions.

$$
\begin{array}{r}
P 2=y_{2}^{d}\left(\rho^{*}, \alpha\right) \\
D P 2=\dot{y}_{2}^{d}\left(\rho^{*}, \alpha\right) \\
D^{2} P 2=\ddot{y}_{2}^{d}\left(\rho^{*}, \alpha\right)
\end{array}
$$

where, $\rho^{*}=t_{c}+t_{s}$ is the designed convergence time which corresponds to the time when the splines smoothly intersects the CWF. Note that the evaluation of the spline 
will be computed from the following equation:

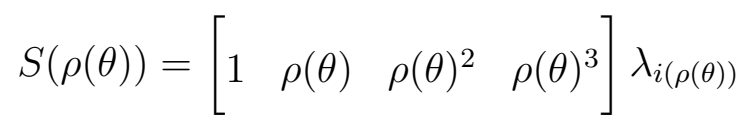

where, $\lambda_{i(\rho(\theta))}$ is the set of spline parameters corresponding to $\rho(\theta)$. The relative degree two outputs will depend on the spline trajectory or the CWF. Therefore, the outputs can be expressed as:

$$
y_{2}(\theta, \alpha)=\left\{\begin{array}{lll}
y_{2}^{a}(\theta)-S(\rho(\theta)), & \text { if } \quad & \rho(\theta) \leq \rho^{*} \\
y_{2}^{a}(\theta)-y_{2}^{d}(\rho(\theta), \alpha), & \text { if } & \rho(\theta)>\rho^{*}
\end{array}\right.
$$

Note that using the spline formulation, we lose the guarantees about stability because the splines trajectories will evolve outside the PHZD surface. However, since the human inherent controller is highly adaptable and robust we assume that the user will remain stable considering the small changes introduced by the spline. Furthermore, the trajectories generated by the use of splines will converge to the provably stable CWF for flat groud walking.

\subsubsection{Generated Trajectories}

Using the optimization problem proposed, a simulation in MATLAB was performed to obtain the resulting trajectories for ankle and knee under a different initial and final condition (Fig. 4.4) based on the designed flat ground trajectories (Fig. 3.4). The optimization was solved using the analytical solution shown in (4.35). Note that both extremes are generated using the spline formulation for the estimation of the behavior of the joints. However, the implementation of the framework dictates that the first part the prosthetic standing phase is generated using the spline trajectory generation and the last part of the prosthetic swing phase is generated through 
the use of low gain PD control. The results achieved using the analytic form of the problem yielded the same results as using the quadratic program solver built-in in MATLAB. This results were also tested in $\mathrm{C}++$ for a series of different conditions to ensure that the solutions are valid.
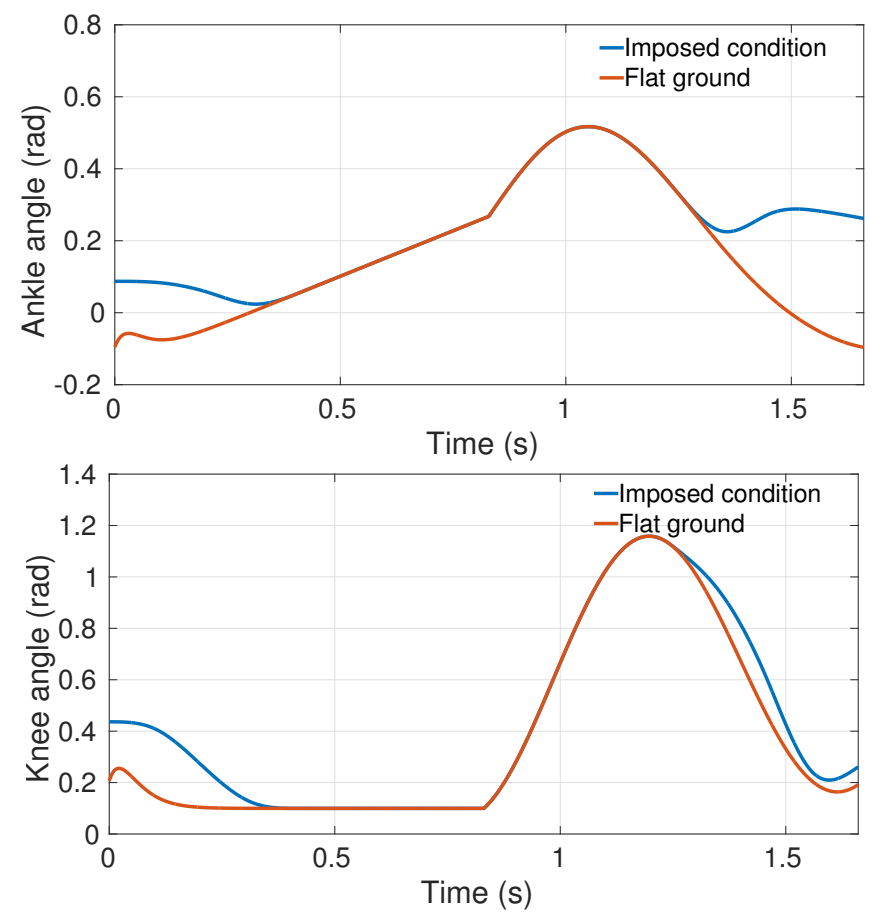

Figure 4.4: Trajectory generation for ankle and knee joint. The splines has been generated in two extremes to show the effect of the splines.

\subsection{Implementation Details}

Table 4.1 shows the parameters used during the implementation of the framework and the time of action of each strategy. The parameterized time indicates $100 \%$ of step progression. The low gain PD control is used on the final part of the prosthetic swing phase $(\rho(\theta) \geq 0.6)$ and is aimed to grant terrain adaptation and provide the next initial conditions for the next step. 
Table 4.1: Implementation parameters

\begin{tabular}{|l|l|l|}
\hline Parameter & Value & Unit \\
\hline Switching time & 0 & Unitless \\
\hline Convergence time & 0.2 & Unitless \\
\hline Impedance time & 0.6 & Unitless \\
\hline Parameterized time (max) & 0.823 & Unitless \\
\hline Number of splines & 4 & Unitless \\
\hline
\end{tabular}

Note that in order to satisfy the smooth conditions for position, velocity and acceleration, it is required to solve 3 equations for each waypoint, obtaining a total of $3(N+1)$ equations. Given that each spline have 4 parameters, the number of variables of the whole trajectory is $4 N$. To find a solution the number of variables must be greater or equal to the number of constraints $4 N \geq 3(N+1)$, resulting in $N \geq 3$. During the current implementation the number of splines selected was $N=4$. Choosing $N=3$ would eliminate the possibility to shape the trajectories through the optimization. 


\section{RESULTS}

In order to verify the applicability of the algorithms proposed they were implemented in the transfemoral prosthesis AMPRO II. The experiments were performed by a healthy subject with a knee adapter [13] (Fig. 5.1) that allowed him to bend his human knee to use the transfemoral prosthesis simulating an amputation. Additionally, a comparison between Human-Inspired Control (HIC) and the Splines-based Human-Inspired Control (SHIC) is realized to motivate the usage of SHIC as a controller able to perform flat ground and upslope walking.

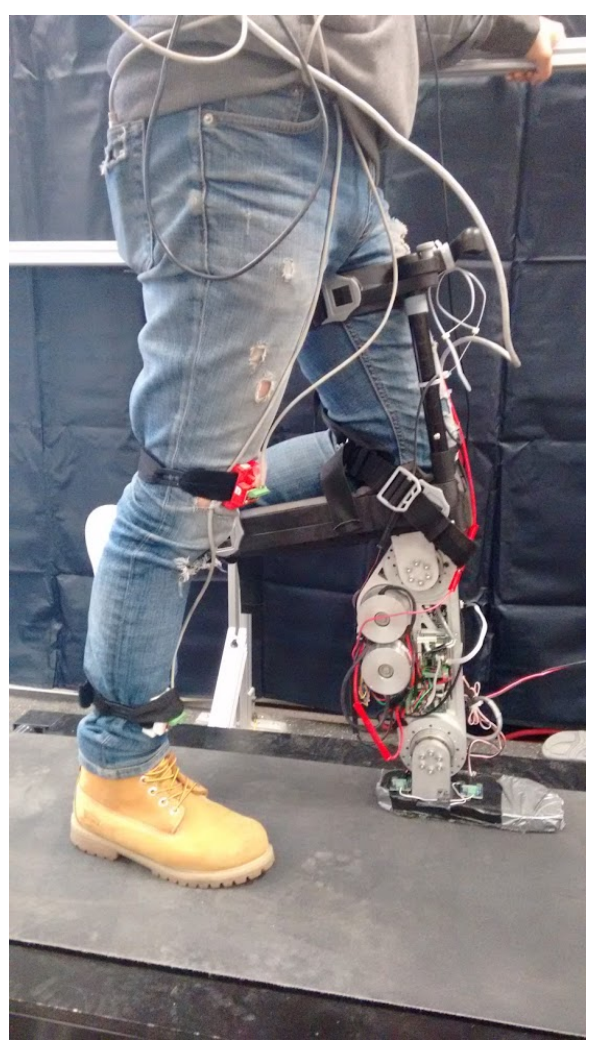

Figure 5.1: Healthy subject using a knee adapter to use the transfemoral prosthesis AMPRO II 


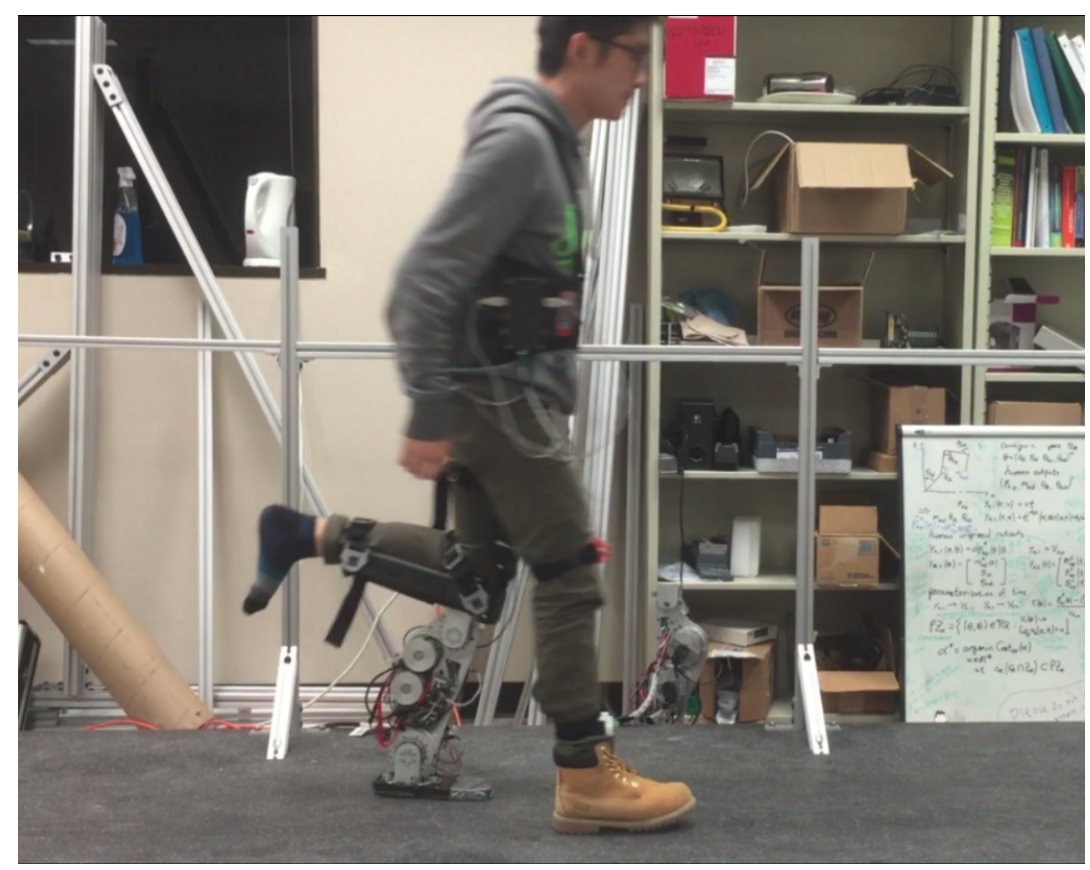

Figure 5.2: Indoors flat ground walking experiment using SHIC.

The experiments were realized inside the laboratory space (Fig. 5.2) and outside of the laboratory (Fig. 5.3). Both experiments consisted in flat ground walking and upslope walking tests. The subject was asked to perform two trials: (1) to walk in straight line in a flat ground surface for $5 \mathrm{~min}$, and (2) walk in flat ground terrain toward an slope and immediately transition into upslope walking without any stop procedure.

\subsection{Flat Ground Walking}

The spline-based controller is aimed to enable an upslope walking, however, it is expected to work even for flat ground terrain. In order to support this claim flat ground experiments were realized using HIC and SHIC under the same conditions and letting the human subject to use a self-paced speed. In both experiments, after some practice the user achieved to walk without holding the security handrails as 


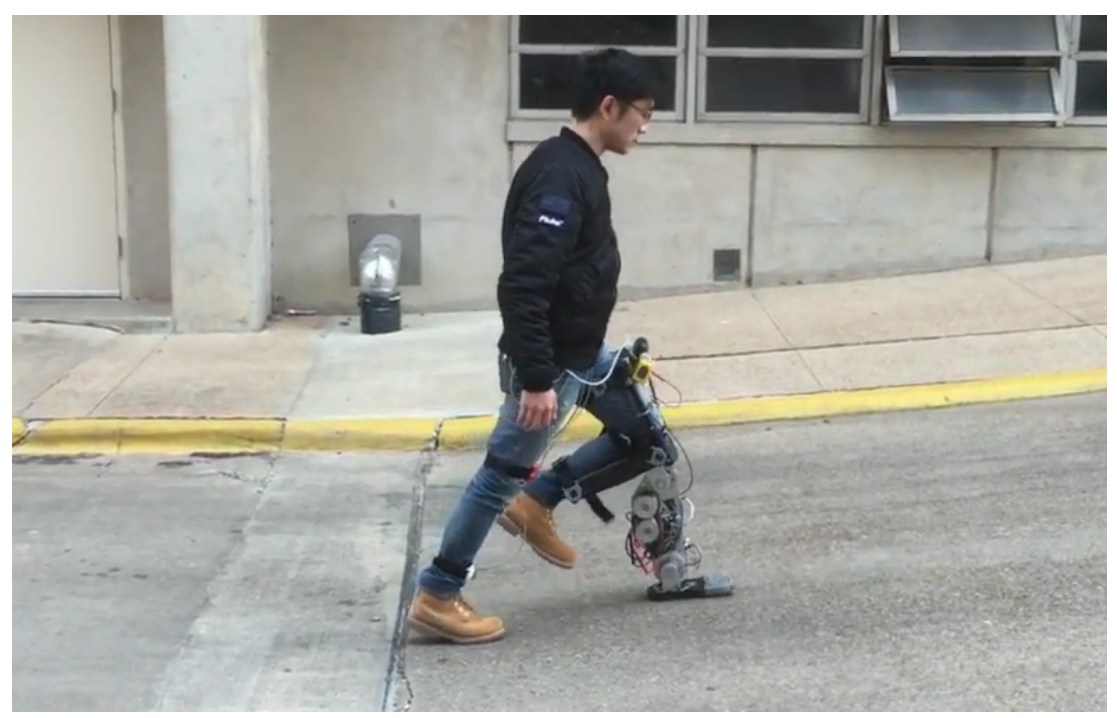

Figure 5.3: Outdoors experiment using SHIC.

seen in Fig. 5.10, which particularly corresponds to SHIC.

\subsubsection{HIC Results}

HIC was used in the AMPRO II and provided stable walking for the subject. The trajectories achieved are shown in Fig. 5.4 for the ankle and knee respectively. Note that tracking is achieved throughout the entire trajectory.
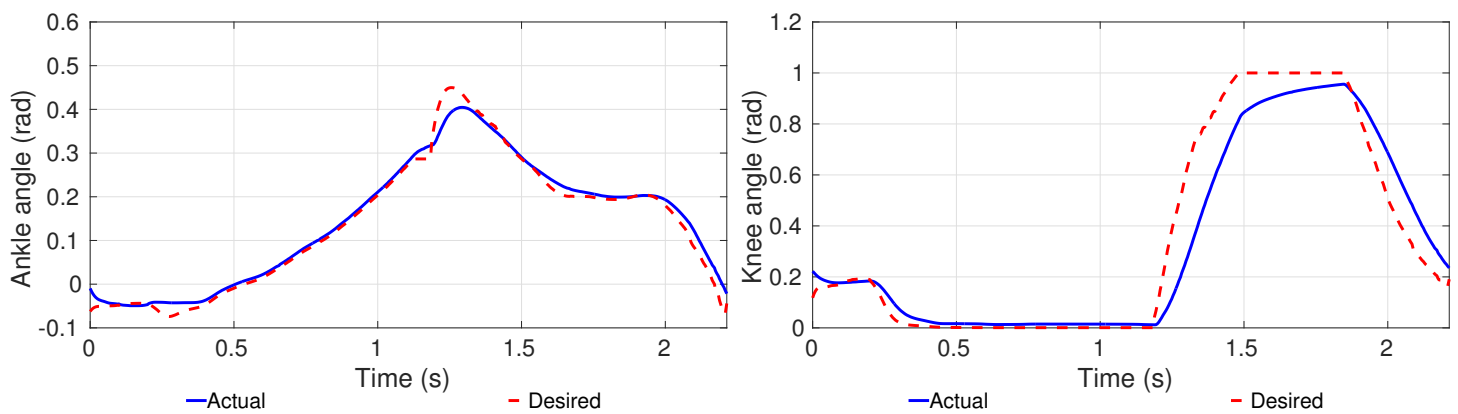

Figure 5.4: Ankle and knee tracking for flat ground walking using HIC. 


\subsubsection{SHIC Results}

SHIC was used in AMPRO II, the experiments lead as well to stable walking. The trajectories achieved are shown in Fig. 5.5 for ankle and knee respectively. Note that tracking is lost in the final part of the step because of the low gain PD.
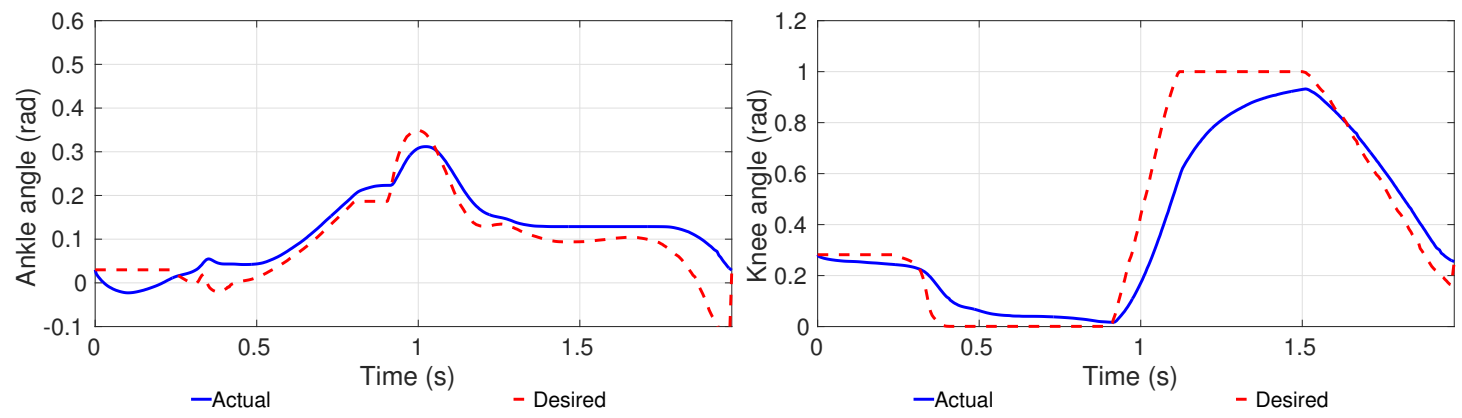

Figure 5.5: Ankle and knee tracking for flat ground walking using SHIC.

\subsection{Upslope Walking}

The main focus of the thesis is to show upslope walking capabilities in the transfemoral prosthesis. The upslope walking data was captured during the indoors experiment (Fig. 5.11), were the subject performed several trials on the sloped surface. The inclination of the slope was $13^{\circ}$. Fig. 5.6 represents the trajectories of the ankle and knee respective.

\subsection{Controllers Comparison}

Both controllers HIC and SHIC resulted in stable walking for flat ground walking, however they have some differences in the trajectories generated. Because of this, it is important to explain the differences in the controller action that leads to such differences. Additionally, a comparison between flat ground walking and upslope 

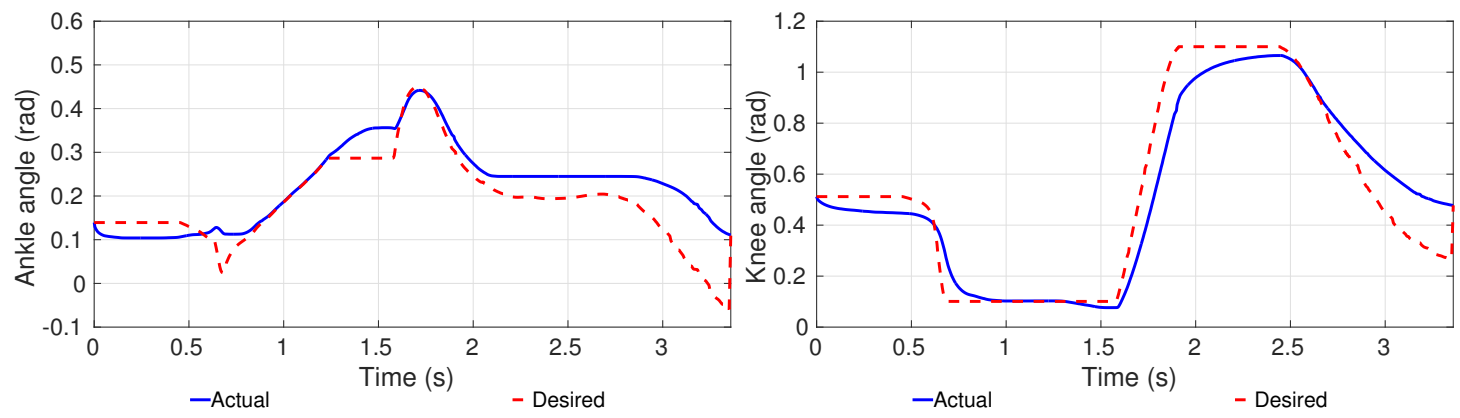

Figure 5.6: Upslope walking trajectories for ankle and knee. The slope inclination is $13^{\circ}$.

walking with SHIC is realized to observe the effect of the splines.

\subsubsection{Flat Ground Walking using HIC and SHIC}

Comparing the trajectories generated by the use of HIC and SHIC (Fig. 5.7) it can be noted that SHIC ankle suffers a deviation with respect to HIC, this is explained by the low gain PD algorithm which is used for terrain adaptation. The knee joints show a very similar evolution for both HIC and SHIC controllers. It is important to recall that both controllers lead to stable walking during the experiments. The SHIC experimental walking tiles can be seen in Fig. 5.10.
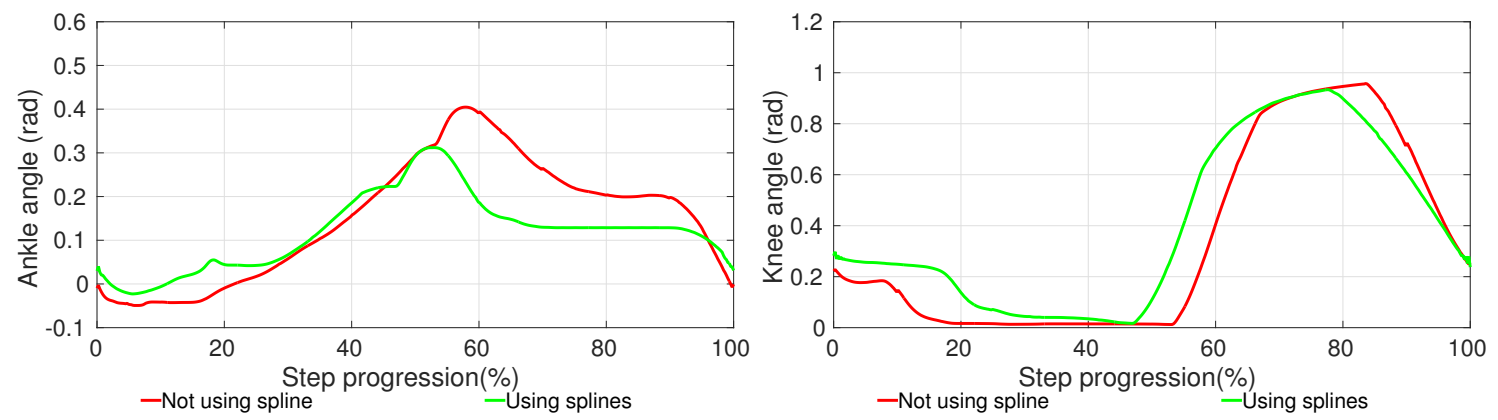

Figure 5.7: Superposition of the trajectories for flat ground surfaces using both controllers: HIC (red) and SHIC (green) 


\subsubsection{Flat Ground and Upslope Walking using SHIC}

By exploring the differences in the trajectories obtained through the use of the splines-based controller is possible to visualize the effect of the SHICs and the verification of the strategies proposed to perform upslope walking. The ankle and knee trajectories are shown in Fig. 5.8 for both terrains. It can be observed that the upslope ankle shows a small offset with respect to flat ground walking, however knee presents the qualitative behavior expected: an increase on knee flexion to avoid foot scuffing and to mimic the human strategies for upslope walking. Note that the assumption of flat foot affects primarily the ankle behavior. The next step considered is the implementation of a multi-contact walking algorithm [31].
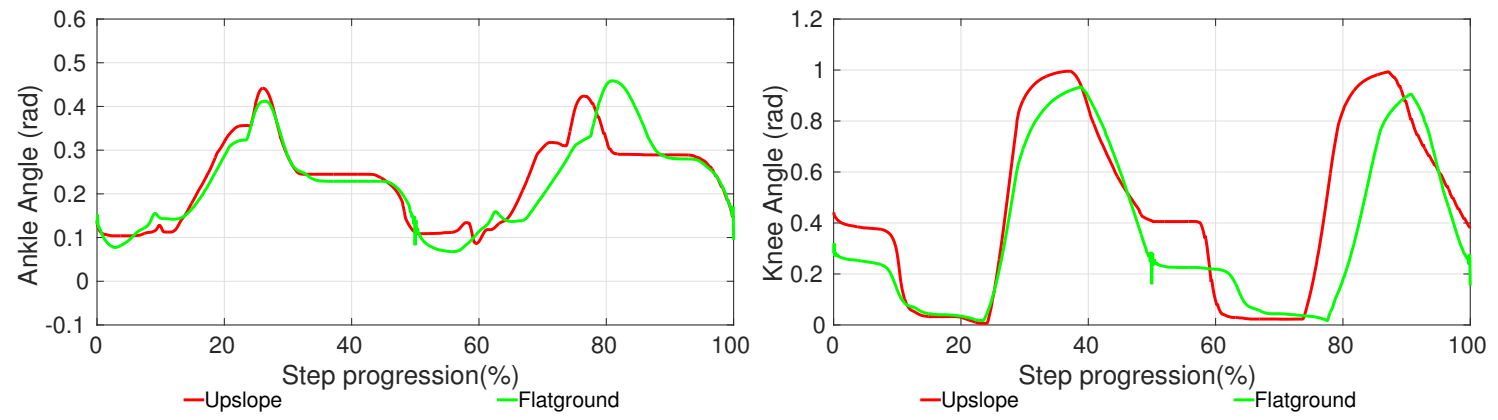

Figure 5.8: 2-steps trajectory differences between flat ground (green) and upslope walking (red) using SHIC.

\subsection{SHIC Control Action}

The control action of the SHIC can be observed by looking at the tracking plots, in particular Fig. 5.9 shows the regions of action of the low gain PD and the splines. In particular, the red shaded region corresponds to the action of the low gain $\mathrm{PD}$, it is observed that in this region the tracking is lost in order to gain terrain adaptation. 
The blue shaded regions corresponds to the action of the splines, which takes the actual value of the joints and blends the trajectory to perform upslope walking.
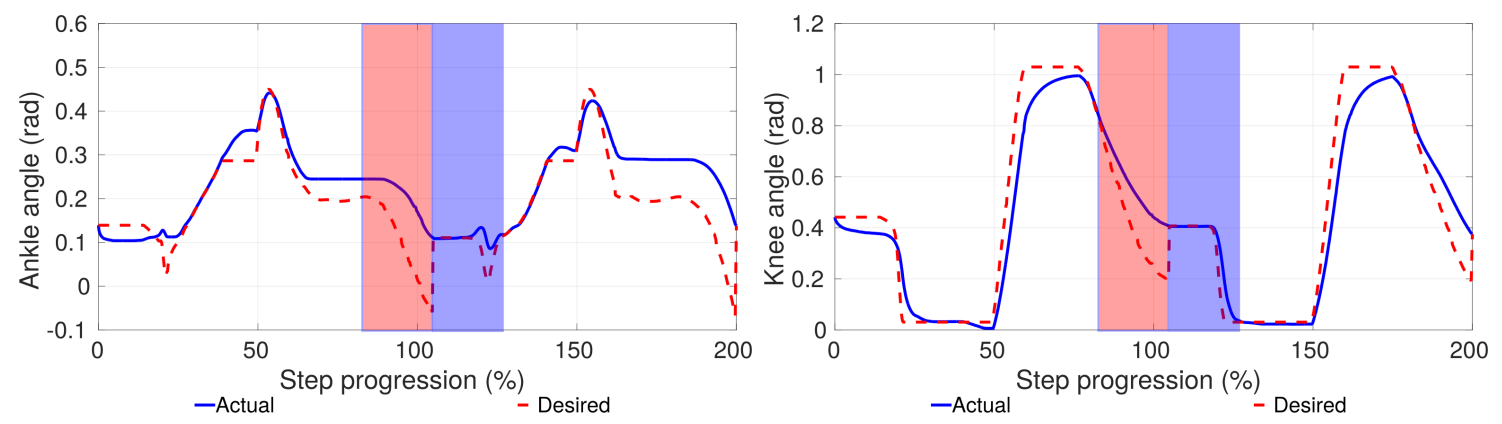

Figure 5.9: SHIC low gain PD (red region) and spline generation (blue region) for two steps. 


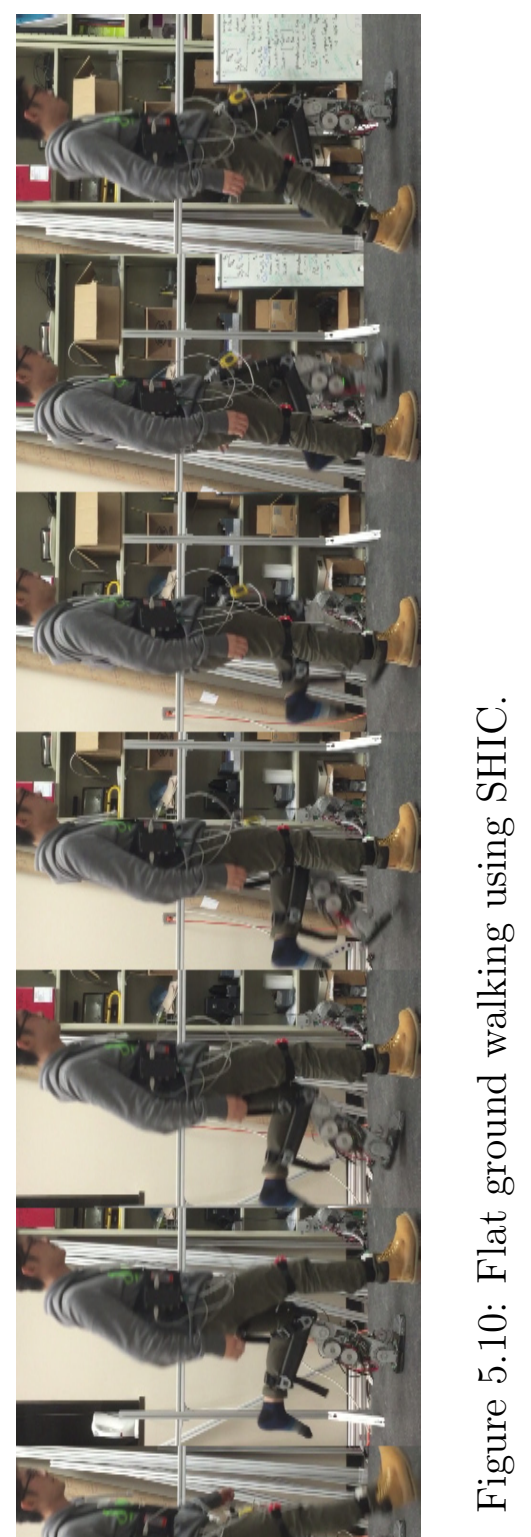




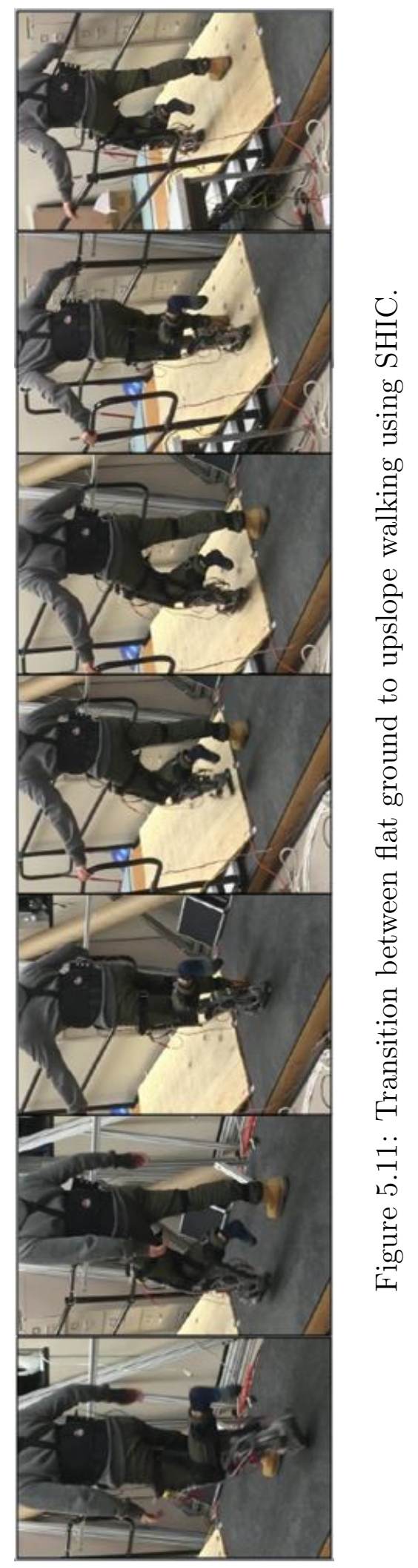




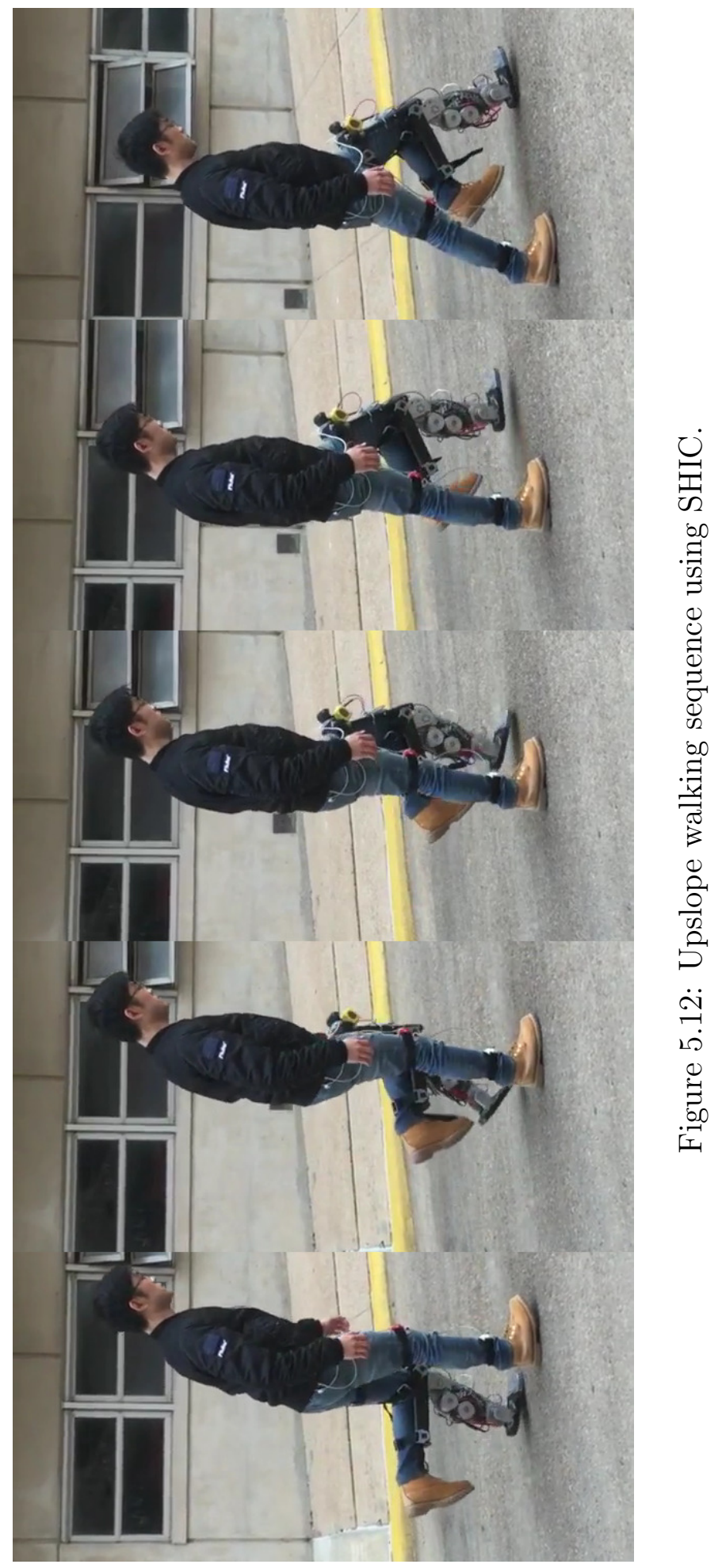




\section{CONCLUSIONS}

The proposed algorithm successfully reproduced the strategy of healthy humans when they perform upslope walking, leading the human subject to perform upslope walking with the prosthetic device AMPRO II. The tiles (Fig. 5.10, Fig. 5.11 and Fig. 5.12) show that the same control algorithm can be used to generate flat ground and upslope walking gaits within the same framework. In fact, the comparison made for flat ground walking using both controllers yielded similar results for ankle and knee (Fig. 5.4) which motivates the use of the SHIC for the generation of gaits for flat ground and upslope walking.

During upslope walking the knee trajectory generated by the use of splines and low gain PD control is consistent with the strategy found during healthy human walking in inclined surfaces $[16,14,12]$, furthermore, the changes in knee are correlated to the human knee sensitivity with respect to the slope inclination [16].

The thesis proposed successfully a control framework based on the use of a set of cubic-splines based on an optimization problem and low gain PD control for performing upslope walking. The control framework blends the joint trajectories from flat ground walking into upslope walking. Furthermore, the controller automatically generates walking gaits for flat ground and upslope walking without large tuning requirements, and allowing a seamless, immediate transition between surfaces.

Future research needs to be focused on the use of multi-contact walking gaits with the set of cubic splines proposed. Additionally, entire trajectories could be generated using an appropriate optimization formulation. 


\section{REFERENCES}

[1] Aaron D. Ames. First steps toward automatically generating bipedal walking from human data. Robot Motion and Control 2011, Springer London:89-116, 2012.

[2] Aaron D. Ames. Human-inspired control of bipedal walking robots. IEEE Transactions on Automatic Control., 59(5), 2014.

[3] B Cao, GI Dodds, and GW Irwin. Constrained time-efficient and smooth cubic spline trajectory generation for industrial robots. In Control Theory and Applications, IEE Proceedings-, volume 144, pages 467-475. IET, 1997.

[4] Rafal Goebel, Ricardo G Sanfelice, and Andrew Teel. Hybrid dynamical systems. Control Systems, IEEE, 29(2):28-93, 2009.

[5] Robert D Gregg and Jonathon W Sensinger. Towards biomimetic virtual constraint control of a powered prosthetic leg. Control Systems Technology, IEEE Transactions on, 22(1):246-254, 2014.

[6] A Hereid, S. Kolathaya, M.S Jones, Van Why J.W, and A. D. Ames. Dynamic multi-domain bipedal walking with atrias through slip based human-inspired control. In ACM, editor, Proceedings of the 17th International conference on Hybrid systems: computation and control., 2014.

[7] Ayonga Hereid, Christian M Hubicki, Eric A Cousineau, Jonathan W Hurst, and Aaron D Ames. Hybrid zero dynamics based multiple shooting optimization with applications to robotic walking. In Robotics and Automation (ICRA), 2015 IEEE International Conference on, pages 5734-5740. IEEE, 2015. 
[8] Neville Hogan. Impedance control: An approach to manipulation: Part iiimplementation. Journal of dynamic systems, measurement, and control, 107(1):8-16, 1985.

[9] Shishir Kolathaya and Aaron D Ames. Achieving bipedal locomotion on rough terrain through human-inspired control. In Safety, Security, and Rescue Robotics (SSRR), 2012 IEEE International Symposium on, pages 1-6. IEEE, 2012.

[10] J. Zico Kolter and Andrew Y. Ng. Task-space trajectories via cubic spline optimization. In Robotics and Automation, 2009. ICRA '09. IEEE International Conference on, pages 1675-1682, 2009.

[11] Brian Edward Lawson, J Mitchell, Don Truex, Amanda Shultz, Elissa Ledoux, and Michael Goldfarb. A robotic leg prosthesis: Design, control, and implementation. Robotics \& Automation Magazine, IEEE, 21(4):70-81, 2014.

[12] Andrea N. Lay, Chris J. Hass, and Robert J. Gregor. The effects of sloped surfaces on locomotion: A kinematic and kinetic analysis. Journal of Biomechanics, 39:1621-1628, 2006.

[13] Edward D Lemaire, David Nielen, and Marie Andrée Paquin. Gait evaluation of a transfemoral prosthetic simulator. Archives of physical medicine and rehabilitation, 81(6):840-843, 2000.

[14] Alain Leroux, Joyce Fung, and Hugues Barbeau. Postural adaptation to walking on inclined surfaces: I. normal strategies. Gait \&ु posture, 15(1):64-74, 2002.

[15] Owings Maria F. and Kozak Lola Jean. Ambulatory and inpatient procedures in the united states, 1996. National Center for Health Statistics., Vital Health(Stat 13(139)), 1998. 
[16] Andrew Stuart McIntosh, Karen T. Beatty, Leanne N. Dwan, and Deborah R. Vickers. Gait dynamics on an inclined walkway. Journal of Biomechanics., 39(13):2491-2502, 2006.

[17] Craig D Murray. The social meanings of prosthesis use. Journal of Health Psychology, 10(3):425-441, 2005.

[18] T.S. Parker and L.O. Chua. Practical numerical algorithms for chaotic systems. Springer New York, 1989.

[19] Shankar. Sastry. Nonlinear systems: analysis, stability, and control., volume 10. Springer Science \& Business Media, 2013.

[20] Ryan W. Sinnet, Matthew J. Powell, Rajiv P. Shah, and Aaron D. Ames. A human-inspired hybrid control approach to bipedal robotic walking. In 18th IFAC World Congress, 2011.

[21] Douglas G. Smith. The transfemoral amputation level, part 1. URL: "http://www.amputee-coalition.org/resources/transfemoral-amputationpart-1/", Accessed: 03/01/2016.

[22] Frank Sup, Amit Bohara, and Michael Goldfarb. Design and control of a powered transfemoral prosthesis. The International Journal of Robotics research., $27(2): 263-273,2008$.

[23] Frank Sup, Huseyin Atakan Varol, and Michael Goldfarb. Upslope walking with a powered knee and ankle prosthesis: initial results with an amputee subject. Neural Systems and Rehabilitation Engineering, IEEE Transactions on., 19(1):71-78, 2011.

[24] RL Waters, J Perry, D Antonelli, and H Hislop. Energy cost of walking of amputees: the influence of level of amputation. The Journal of bone and joint 
surgery., 1(58):42-46, 1976.

[25] Eric DB Wendel and Aaron D. Ames. Rank properties of poincare maps for hybrid systems with applications to bipedal walking. In ACM, editor, Proceedings of the 13th ACM international conference on Hybrid systems: computation and control., 2010.

[26] E. R Westervelt, J. W. Grizzle, C Chevallereau, J Choi, and B. Morris. Feedback control of dynamic bipedal robot locomotion. CRC press, 2007.

[27] E.R Westervelt, J.W Grizzle, and Koditscheck. D.E. Hybrid zero dynamics of planar biped walkers. IEEE Transactions on Automatic Control, 48(1):42-56, 2003.

[28] Shishir Nadubettu Yadukumar, Murali Pasupuleti, and Aaron D. Ames. From formal methods to algorithmic implementation of human inspired control on bipedal robots. In Springer Berlin Heidelberg, editor, Algorithm Foundations of human inspired control on bipedal robots., pages 511-526, 2013.

[29] H. Zhao, J. Reher, J. Horn, V. Paredes, and A. D. Ames. Realization of stair ascent and motion transitions on prostheses utilizing optimization-based control and intent recognition. In IEEE, editor, Rehabilitation Robotics (ICORR), 2015 IEEE International Conference on., 2015.

[30] Hui-Hua Zhao, WL Ma, Zeagler MB, and A. D. Ames. Human-inspired multicontact locomotion with amber2. ACM/IEEE 5th International Conference on Cyber-Physical Systems (with CPS Week 2014), IEEE Computer Society, 2014.

[31] Huihua Zhao, Jonathan Horn, Jacob Reher, Victor Paredes, and Aaron D Ames. Multi-contact locomotion on transfemoral prostheses via hybrid system models and optimization-based control. 
[32] Huihua Zhao, M. J. Powell, and A. D. Ames. Optimizal Control Applications and Methods, chapter Human-inspired motion primitives and transitions for bipedal robotic locomotion in diverse terrain., pages 730-755. 2014.

[33] Huihua Zhao, Jake Reher, Jonathan Horn, Victor Paredes, and Aaron D. Ames. Realization of nonlinear real-time optimization based controllers on self-contained transfemoral prosthesis. In ACM, editor, Proceedings of the ACM/IEEE Sixth International Conference on Cyber-Physical Systems., 2015. 